\title{
Beziehungen zwischen der entwicklungshemmenden Wirkung des Vollbluts auf Tuberkelbazillen und der Komplementbindungsreaktion.
}

\author{
Von \\ Shigeyoshi Akanuma. \\ (赤沼茂 芳) \\ (Aus der medizinischen Kliniti von Prof. T. Kumagai, \\ Tohohu-Universität zu Sendai.)
}

\section{Einleitung.}

Es ist allgemein bekannt, dass bei der Infektion von Menschen und Tieren mit Tuberkelbazillen Tuberkulinallergie entsteht, indem im Serum der infizierten Antikörper wie Bakteriolysin, Opsonin, Praecipitin, Agglutinin, Amboceptor der Komplementbindung usw. auftreten. Es ist aber noch nicht klargestellt, wieviel diese Antikörper mit dem Schutz Tuberkulöser gegen erneute Injektion zu tun. laben, jedenfalls aber stehen sie in sehr innigem Verhältnis zur Immunität.

Wrigh $\mathrm{t}^{1}$ hat im Jahre 1924 eine neue Methode erdacht, mit Hilfe von "Capillary Tubes" und ,Slide Cells" die Kultur der Tuberkelbazillen im Vollblut zu züchten. Mit Hilfe dieser Methode hat er gefunden, dass im Blut Tuberkulöser das Wachstnm der Tuberkelbazillen stärker gehemmt wird als in dem gesunder Erwachsener. Es sieht hierbei die entwicklunghemmende Wirkung auf Tuberkelbazillen nicht im Plasma, sondern in der Phagozytose der Leukozyten.

Darauf hat Banner man $n^{2}$ bei bovinen Tuberkelbazillen gefunden, dass zwischen dem Plasma Tuberkulöser und dem Gesunden ein deutlicher Unterschied im Wachstum besteht. Meissner ${ }^{3)}$ hat auch unter Gesunden individuelle Unterschiede im Wachstum des'Tuberkelbazillus im Vollblut gefunden.

Sonak $k^{4}$ hat das Verhältnis des Wachstums von Tuberkelbazillen im Vollblut zur Tuberkulinallergie verfolgt und berichtet, dass das Wachstum bei tuberkulinpositiven Kindern viel stärker als bei tuberkulinnegativen gehemmt wird. 
Sato $0^{53}$ hat mit Hilfe der Wrightschen Methode gefunden, dass es einen Unterschied im Wachstum der Tuberkelbazillen im Vollblut und Plasma zwischen gesunden und mit Tuberkelbazillen immunisierten Meerschweinchen gibt, dass aber in der Hemmung des Wachstums der Bazillen kein bedeutender Unterschied zwischen Vollblut und Plasma besteht und Leukozyten keinen Einfluss auf das Wachstum der Bazillen ausüben. It ${ }^{6}$ hat an Meerschweinchen die Zeitdauer bis zum Auftritt der Hemmungswirkung nach Tuberkuloseinfektion verfolgt; er fand, dass bei der Impfung mit Tuberkelbazillen diese im wesentlichen parallel mit der Tuberkulinallergie auftritt.

Darnach haben $\mathrm{Og}$ at a und Schibuka wa ${ }^{7)}$ mitgeteilt, dass es bei gesunden Erwachsenen individuelle Unterschiede im Wachstum der Bazillen gibt und dass ihr Wachstum bei tuberkulinpositiven viel stärker als bei negativen gehemmt wird, womit sie die oben erwähnten Mitteilungen von Meissner sowie von Sonak bestätigten.

Ferner haben Imamura und Schibuka wa ${ }^{8 ; 9}$ an Tuberkulösen in verschiedenen Krankheitsstadien die Beziehung zwischen der entwicklunghemmenden Wirkung des Vollbluts auf Tuberkelbazillen und der Tuberkulinallergie verfolgt und dabei festgestellt, dass die hemmende Wirkung immer parallel mit der Tuberkulinallergie verläuft und dazu neigt, mit dem Fortschreiten der Krankheit allmählich stärker zu werden, dass aber die Bazillenentwicklung wieder lebhaft wie bei Gesunden wird, wenn die Krankheit ins schwere terminale Stadium ubergeht. Ogata ${ }^{10)}$ hat beim Tierversuch festgestellt, dass Hunger, Hyperglykämie, Anämie usw. günstig auf das Wachstum der Tuberkelbazillen im Vollblut ein wirken und dass die Röntgenbestrahlung sie eine Weile hemmt, aber danach wieder fördert.

Aus Obigem ist zu ersehen, dass die entwicklunghemmende Wirkung auf die Tuberkelbazillen des Vollbluts wahrscheinlich im Plasma selbst liegt und bei Tuberkulösen stärker als bei Gesunden auftritt, obwohl unter Gesunden individuelle Unterschiede bestehen. Ferner sieht man, dass die entwicklunghemmende Wirkung gleichzeitig mit der Entstehung der Tuberkulinallergie auftritt und mit dem Fortschreiten der Stadien sowie der Tuberkulinallergie zwar immer parallel verläuft, dass jedoch das Bazillenwachstum im Vollblut wieder lebhaft wird, wenn die Krankheit sehr schwer wird und die Tuberkulinreaktion sich der $\mathrm{H}$ ajek schen negativen Anergie nähert. Diese entwicklunghemmende Wirkung des Vollbluts auf Tuberkelbazillen steht also in einem gewissen Verhältnis zur Tuberkuloseimmunität und lässt sich auf irgend einen Immunstoff im Plasma zurückführen. 
Dann erhebt sich die Frage, ob zwischen ihr und den verschiedenen Antikörper'n im Serum irgend eine nahe Beziehung besteht. Hierüber ist bisher noch nichts veröffentlicht worden. Zur Klärung dieser Frage haben wir deshalb vorliegende Versuche ausgeführt.

Als Methoden, die Tuberkuloseimmunität biologisch festzustellen, sind Komplementbihdungsreaktion, Aggultination, Präzipitation usw. angewandt worden. Dass die erste unter all diesen eine hervorragende Stellung einnimmt, wird von allen Seiten anerkannt. Jhr klinischer Wert ist auch durch folgende Mitteilungen aus unserer Klinik betont worden : 1932 verfolgte Sasak i ${ }^{11}$ den Verlauf und das Sclicksal der Komplementbindungsreaktion bei Tuberkulösen, 1933 berichteten Kumagai und Ozeki, ${ }^{\left.1{ }^{2}\right)}$ dass der Verlauf und die Prognose der Krankheit bei Lungentuberkulösen durch dauernde Beobachtung dieser Reaktion zusammen mit der Tuberkulinallergie der Haut festgestellt werden können. Ich richtete mein Augenmerk darauf, festzustellen, ob ein gewisser Zusammenhang zwischen der entwicklunghemmenden Wirkung des Vollbluts und der Komplementbindungsreaktion besteht, und untersuchte ferner aufs neue die Beziehung der entwicklunghemmenden Wirkung des Vollbluts zu Krankheitsstadium, Tuberkulinallergie, Körpertemperatur, Senkungsreaktion der Blutkörperchen, Leukozytenzahl und Lymphozytenprozentsatz. Im folgenden möchte ich meine diesbezüglichen Ergebnisse mitteilen.

\section{Methode.}

Die Gesamtzahl der untersuchten Patienten betrug 161 (Primärinfektion, Pleuritis sowie Peritonitis 37, gebesserte Fälle von Frühinfiltrat und Lungentuberkulose 104, gestorbene Fälle von Lungentuberkulose 20), die in unsere Klinik aufgenommen und längere Zeit hindurch behandelt worden sind. Bei ein und demselben Kranken wurden mit Zwischenpausen von 1-3 Monaten der Zustand des Wachstums der Tuberkelbazillen im Vollblut und seine Schwankungen beobachtet.

Als Versuchsmethode wurde die Wrightsche „Slide Cell Culture“ benutzt. Ich nahm als Kultur die Tuberkelbazillen des humanen Typus „S-Stamm," der anch als Antigen der Komplementbindungsreaktion verwandt worden war, und zwar eine frische und 20 Tage lang auf Gingko-Nährboden nach Su zu ki ${ }^{13}$ gezüchtete Kultur. Um eine vollkommen homogenisierte Bazillenaufschwemmung zu bekommen, verfuhr ich folgendermassen: 5 Ösen Tuberkelbazillen wurden in einen durch Alkohol und Äther desinfizierten und dann getrockneten Achatmörser getan und mit $10 \mathrm{ccm}$ sterilisierter physiologischer Kochsalzlösung sorgfältig zerrieben. Zur Entfernung gröberer Partikel wurde eine halbe Stunde 
lang mit der Zentrifuge bei einer Umdrehungszahl von etwa 3000 pro Minute zentrifugiert. Dann entnahm man mit steriler Pipette der dem Bodensatz nächsten mittleren Schicht vorsichtig $3 \mathrm{ccm}$ Lösung.

Mit einer Injektionsspritze wurde der Kubitalvene der Patienten $1 \mathrm{ccm}$ Blut entnommen und mittels einer Pipette mit $0,1 \mathrm{ccm}$ der auf die Reagensgläser verteilten Bazillenaufschwemmung schnell und gut gemischt und das Gemisch dam in die ,Slide Cells" gegossen. Als Kontrolle wurde das Blut sog. Tuberkulosefreier verwandt, deren Körpertemperatur normal, Tuberkulinreaktion sowie Komplementbindungsreaktion negativ, und Senkungsreaktion der Blutkörperchen normal waren und deren röntgenologischer Lungenbefund keine pathologische Veränderung zeigte.

Wright fand bei den 24 Stunden lang kultivierten $P_{1}$ äparaten, bis zu 2 bezw. 5 Stück entwickelte Bazillenkolonien, die nach weiteren 48 Stunden so gross wurden, dass sie bei schwacher Vergrösserung mikroskopisch sichtbar wurden. Aber bei meinem Versuch mit dem S-Stamm nahmen die Bazillen unmittelbar nach Kultivierung zwar Stäbchengestalt wie gewöhnliche Tuberkelbazillen an, aber nach 2tägiger Kultivierung wurden sie zu punkt- oder staubförmigen Stückchen ohne Säurefestigkeit, so dass sie nicht wie Tuberkelbazillen aussahen; erst nach 3 Tagen nahmen sie die Gestalt diplo- oder streptokokkenartiger Bazillen an, und nach 4 Tagen ballten sie sich zu als 2-10 Stückchen entwickelten Tuberkelbazillen zusammen.

Diese Erscheinung stimmt mit dem schon von Marmorek, ${ }^{14)}$ Sp e n gle $r,{ }^{15}$, $\left.\left.\mathrm{Much}{ }^{16)} \mathrm{Knoll}^{17}{ }^{17}\right) \mathrm{Kahn}^{18}\right) \mathrm{Spanie}^{199}$ n. a. erwähnten Vorgang übereill, wonach sich die Tuberkelbazillen durch Phagozytose der Leukozyten in nicht säurefeste Körner verwandeln und sich dann zu Stäbchen entwickeln. Deshalb. wurde bei unserem Versuch die Kontrolle 5 mal, d. h. nach 2, 4, 6, 8 und $10 \mathrm{Ta}$ gen, ansgeführt, die jedesmal mit den Tuberkelbazillen im Vollblut der Untersuchten verglichen wurde, wobei die, welche dem Präparate der Kontrolle von: je $2,4,6,8$ und 10 Tagen entsprechen, als Bazillenentwicklung $(-),(+),(+t),(\mathrm{Ht})$ und (IHI) bezeichnet wurden; $(-)$ und $(+)$ bedeuten: fast gar nicht oder schlecht entwickelt, (tt) und (Ht) ziemlicht gut und (HII) sehr gut entwickelt. Zur Färbung wurde Ziehl-Pikrinsäure nach J ötten urd dem Ha a rma n nschen Verfahren ${ }^{20}$ verwandt.

\section{Versuchsergebnisse.}

Nach Kumagai ${ }^{21)}$ kann man die Stellung der Diagnose oder wieder Prognose auf Lungentuberkulose in eine statische, d. h. die der Zustandsbilder, und eine dynamische, d. h. Verfolgung der veränderlichen Krankheitsbilder während einer gewissen Zeitspanne, einteilen. Er betont dabei, dass die ,dynamische“ Beobachtung bei tuberkulösen Erkrankungen sehr wichtig ist. Deshalb haben wir bei unserer Untersuchung sowohl ,statisch" als auch ,dynamisch" beobachtet.

Unsere Versuchsergebnisse in bezug auf das Verhältnis zwischen. 
Tabelle I.

„Dynamische" Beobachtungen der entwicklunghemmenden Wirkung. bei gebesserte Füllen Lungentuberkulöser.

\begin{tabular}{|c|c|c|c|c|c|c|c|c|c|c|}
\hline$\stackrel{+}{\not{z}}$ & 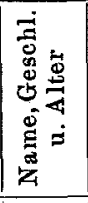 & Diagnose & Datum & 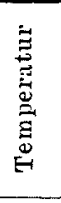 & 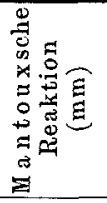 & 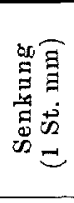 & 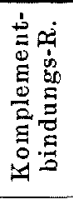 & 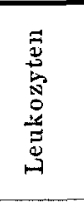 & 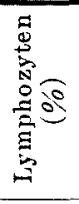 & 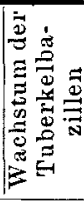 \\
\hline 1 & $\begin{array}{c}\text { C.I. } \\
\text { 9 } \\
21 \mathrm{j} .\end{array}$ & $\begin{array}{l}\text { Primär- } \\
\text { infektion }\end{array}$ & $\begin{array}{l}\text { 10. XI. } \\
\text { 8. XII. } \\
\text { 8. I. } \\
\text { 8. III. }\end{array}$ & $\begin{array}{l}6,8 \\
6,9 \\
7,0 \\
7,2\end{array}$ & $\begin{array}{cc}0 \\
4 \times 4 \\
4 \times 4 \\
4 \times 6\end{array}$ & $\begin{array}{l}68 \\
60 \\
61 \\
97\end{array}$ & $\begin{array}{l}(-) \\
(-) \\
(-) \\
(-)\end{array}$ & $\begin{array}{l}5200 \\
6100 \\
6200 \\
8000\end{array}$ & $\begin{array}{l}42,0 \\
35,0 \\
39,0 \\
22,5\end{array}$ & $\begin{array}{l}(H H) \\
(H H) \\
(H H) \\
(H+)\end{array}$ \\
\hline 2 & $\begin{array}{c}\text { K.K. } \\
\text { of } \\
7 \text { j. }\end{array}$ & $\begin{array}{l}\text { Primär- } \\
\text { infektion }\end{array}$ & $\begin{array}{l}\text { 13. IX. } \\
\text { 11. XII. } \\
\text { 17. I. }\end{array}$ & $\begin{array}{l}7,3 \\
t, 8 \\
6,7\end{array}$ & $\begin{array}{r}0 \\
0 \\
5 \times 7\end{array}$ & $\begin{array}{l}33 \\
10 \\
30\end{array}$ & $\begin{array}{l}(-) \\
(-)\end{array}$ & $\begin{array}{l}8530 \\
9200 \\
6800\end{array}$ & $\begin{array}{l}30,0 \\
43,0 \\
36,0\end{array}$ & $\begin{array}{l}(H W H) \\
(H+H) \\
(H W)\end{array}$ \\
\hline 3 & $\begin{array}{c}\text { T.M. } \\
\text { ô } \\
25 . \mathrm{j} .\end{array}$ & $\begin{array}{c}\text { Primär- } \\
\text { infektion } \\
\text { mit } \\
\text { Diabetes } \\
\text { mellitus }\end{array}$ & $\begin{array}{l}\text { 11. XII. } \\
\text { 16. III. } \\
\text { 31. V. }\end{array}$ & $\begin{array}{l}7,6 \\
7,0 \\
6,7\end{array}$ & $\begin{array}{c}40 \\
31 \\
28 \times 30\end{array}$ & $\begin{array}{l}45 \\
45 \\
10\end{array}$ & $\begin{array}{l}(-) \\
(-) \\
+1\end{array}$ & $\begin{array}{l}8000 \\
9100 \\
6200\end{array}$ & $\begin{array}{l}25,5 \\
25,0 \\
32,0\end{array}$ & $\begin{array}{l}(\mathrm{HH}) \\
(\mathrm{HH}) \\
(\mathrm{HH})\end{array}$ \\
\hline 4 & $\begin{array}{c}0 . \mathrm{T} \\
\stackrel{+}{+} \\
20 \mathrm{j} .\end{array}$ & $\begin{array}{c}\text { Primär- } \\
\text { infektion }\end{array}$ & $\begin{array}{l}\text { 11. XII. } \\
\text { 8. III. }\end{array}$ & $\begin{array}{l}7,1 \\
6,8\end{array}$ & $\begin{array}{c}50 \\
32 \times 40\end{array}$ & $\begin{array}{l}45 \\
45\end{array}$ & $(-)$ & $\begin{array}{l}9500 \\
8600\end{array}$ & $\begin{array}{l}26,0 \\
38,0\end{array}$ & $\begin{array}{l}(H H) \\
(H+)\end{array}$ \\
\hline 5 & $\begin{array}{c}\mathrm{H.M} . \\
\text { ํ. } \\
23^{j} j\end{array}$ & Plenritis & $\begin{array}{l}\text { 8. XII. } \\
\text { 8. III. }\end{array}$ & $\begin{array}{l}7,0 \\
6,8\end{array}$ & $\begin{array}{l}30 \times 35 \\
30 \times 43\end{array}$ & $\begin{array}{l}45 \\
24\end{array}$ & $\begin{array}{l}(+) \\
(-)\end{array}$ & $\begin{array}{r}10000 \\
8600\end{array}$ & $\begin{array}{l}22,5 \\
37,0\end{array}$ & $\begin{array}{l}(H t) \\
(+)\end{array}$ \\
\hline 6 & $\begin{array}{c}\text { K.H. } \\
\text { 우 } \\
21^{j} \mathrm{j} .\end{array}$ & $\begin{array}{l}\text { Tub. } \\
\text { pulm. } \\
\text { duplex }\end{array}$ & $\begin{array}{l}\text { 30. X. } \\
\text { 29. XII. } \\
\text { 16. IV. } \\
\text { 9. VI. } \\
\text { 13. VIII. } \\
\text { 13. IX. } \\
\text { 11. XI. }\end{array}$ & $\begin{array}{l}7,0 \\
7,0 \\
7,1 \\
7,1 \\
7,2 \\
7,1 \\
6,9\end{array}$ & $\begin{array}{l}20 \times 20 \\
22 \times 25 \\
27 \times 30 \\
17 \times 18 \\
15 \times 20 \\
25 \times 30 \\
13 \times 15\end{array}$ & $\begin{array}{l}43 \\
78 \\
49 \\
62 \\
65 \\
37 \\
37\end{array}$ & $\begin{array}{l}(t+) \\
(H+t) \\
(t+) \\
(H+t) \\
(t+) \\
(H+) \\
(H+) \\
(H)\end{array}$ & $\begin{array}{l}5400 \\
5300 \\
9800 \\
7600 \\
6300 \\
7000 \\
7100\end{array}$ & $\begin{array}{l}31,0 \\
27,5 \\
21,0 \\
11,5 \\
42,0 \\
23,0 \\
25,0\end{array}$ & $\begin{array}{l}(t+) \\
(+) \\
(-) \\
(+) \\
(+) \\
(+) \\
(+)\end{array}$ \\
\hline 7 & $\begin{array}{c}\text { E.K. } \\
\text { 우 } \\
45 \text { i. }\end{array}$ & $\begin{array}{c}\text { Tub. } \\
\text { pulm. } \\
\text { duplex } \\
\text { mit } \\
\text { Largngi- } \\
\text { tis Tbe. }\end{array}$ & $\begin{array}{l}\text { 8. III. } \\
9 . \text { VI. } \\
\text { 13. IX. } \\
11 . \text { XI. } \\
\text { 15. II. }\end{array}$ & $\begin{array}{l}6,9 \\
6,6 \\
6,7 \\
7,0 \\
6,7\end{array}$ & $\begin{array}{l}20 \times 21 \\
20 \times 20 \\
20 \times 20 \\
20 \times 25 \\
30 \times 37\end{array}$ & $\begin{array}{l}95 \\
52 \\
46 \\
70 \\
75\end{array}$ & $\begin{array}{l}(-) \\
(+t) \\
(+) \\
(-)\end{array}$ & $\begin{array}{r}10050 \\
10600 \\
7500 \\
7000 \\
9200\end{array}$ & $\begin{array}{l}14,0 \\
22,0 \\
12,0 \\
15,0 \\
23,0\end{array}$ & $\begin{array}{l}(\mathrm{H}) \\
(+) \\
(+) \\
(+t) \\
(+)\end{array}$ \\
\hline 8 & $\begin{array}{c}\text { I.U. } \\
\text { के } \\
10 \mathrm{j} .\end{array}$ & $\begin{array}{l}\text { Tub. } \\
\text { pulm. } \\
\text { duplex }\end{array}$ & $\begin{array}{l}\text { 19. XI. } \\
12 . \mathrm{I} . \\
\text { 16. III. } \\
\text { 9. VI. } \\
\text { 13. VIII. }\end{array}$ & $\begin{array}{l}7,4 \\
6,8 \\
6,4 \\
6,4 \\
6,4\end{array}$ & $\begin{array}{l}20 \times 20 \\
30 \times 40 \\
30 \times 30 \\
30 \times 30 \\
40 \times 50\end{array}$ & $\begin{array}{l}67 \\
40 \\
40 \\
22 \\
20\end{array}$ & $\begin{array}{l}(-) \\
(-) \\
(-) \\
(-)\end{array}$ & $\begin{array}{l}6100 \\
9600 \\
8900 \\
8900 \\
5800\end{array}$ & $\begin{array}{l}13,0 \\
29,0 \\
21,5 \\
43,0 \\
41,5\end{array}$ & $\begin{array}{l}(-) \\
(-) \\
(t+) \\
(+t) \\
(H+)\end{array}$ \\
\hline 9 & $\begin{array}{c}\text { S.S. } \\
\hat{o} \\
\mathbf{3 7} \mathrm{j} .\end{array}$ & $\begin{array}{l}\text { Tub. } \\
\text { pulm. } \\
\text { duplex }\end{array}$ & $\begin{array}{l}\text { 10. XI. } \\
29 . \text { XII. } \\
\text { 8. III. } \\
\text { 7. VI. } \\
\text { 13. VIII. }\end{array}$ & $\begin{array}{l}6,5 \\
6,5 \\
6,8 \\
6,5 \\
6,4\end{array}$ & $\begin{array}{l}25 \times 25 \\
13 \times 13 \\
15 \times 15 \\
18 \times 18 \\
23 \times 25\end{array}$ & $\begin{array}{r}28 \\
19 \\
8 \\
12 \\
9\end{array}$ & $\begin{array}{l}(+1) \\
(-) \\
(-) \\
(++)\end{array}$ & $\begin{array}{l}8300 \\
9300 \\
6500 \\
4600 \\
7400\end{array}$ & $\begin{array}{l}25,0 \\
29,0 \\
24,5 \\
25,0 \\
26,0\end{array}$ & $\begin{array}{l}(-) \\
(+) \\
(+t) \\
(+H) \\
(+)\end{array}$ \\
\hline
\end{tabular}




\begin{tabular}{|c|c|c|c|c|c|c|c|c|c|c|}
\hline $\begin{array}{l}\vec{z} \\
\vec{z} \\
\vec{z} \\
\overrightarrow{z=1}\end{array}$ & 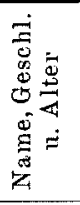 & Diagnose & Datum & 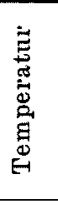 & 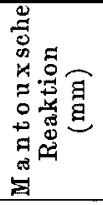 & 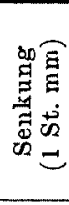 & 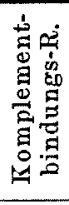 & 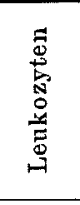 & 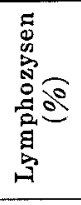 & 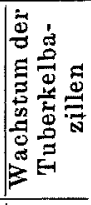 \\
\hline 10 & $\begin{array}{c}\text { T.S. } \\
\text { o } \\
26 \mathrm{j} .\end{array}$ & $\begin{array}{l}\text { Tub. } \\
\text { pulm. } \\
\text { duplex }\end{array}$ & $\begin{array}{l}\text { 30. X. } \\
\text { 12. I. } \\
\text { 8. IV. } \\
\text { 13. VIII. }\end{array}$ & $\begin{array}{l}7,0 \\
8,3 \\
6,9 \\
6,6\end{array}$ & $\begin{array}{l}30 \times 25 \\
30 \times 35 \\
37 \times 40 \\
25 \times 30\end{array}$ & $\begin{array}{r}86 \\
110 \\
68 \\
58\end{array}$ & $\begin{array}{l}(-) \\
(+) \\
(+W)\end{array}$ & $\begin{array}{l}7600 \\
9000 \\
6800 \\
8000\end{array}$ & $\begin{array}{l}14,5 \\
25,0 \\
33,0 \\
29,0\end{array}$ & $\begin{array}{l}(+H) \\
(+) \\
(+n) \\
(-)\end{array}$ \\
\hline 11 & $\begin{array}{c}\text { Y.F. } \\
\stackrel{9}{16 \mathrm{j}} .\end{array}$ & $\begin{array}{l}\text { Tub. } \\
\text { pulm. } \\
\text { duplex }\end{array}$ & $\begin{array}{l}\text { 8. III. } \\
\text { 19. V. } \\
\text { 11. IX. } \\
\text { 17. I. }\end{array}$ & $\begin{array}{l}7,0 \\
6,8 \\
6,6 \\
6,5\end{array}$ & $\begin{array}{l}35 \times 41 \\
30 \times 40 \\
32 \times 35 \\
40 \times 45\end{array}$ & $\begin{array}{l}48 \\
60 \\
32 \\
44\end{array}$ & $\begin{array}{l}(-) \\
(-) \\
(H+H) \\
(H+H)\end{array}$ & $\begin{array}{l}8900 \\
7800 \\
6800 \\
6200\end{array}$ & $\begin{array}{l}20,5 \\
36,0 \\
37,0 \\
38,5\end{array}$ & $\begin{array}{l}(t+t) \\
(t+t) \\
(t+) \\
(t+)\end{array}$ \\
\hline 12 & \begin{tabular}{c} 
S.K. \\
\multirow{\delta}{*}{} \\
$46, \mathrm{j}$
\end{tabular} & $\begin{array}{l}\text { Tub. } \\
\text { pulm. } \\
\text { duplex }\end{array}$ & $\begin{array}{l}\text { 3. XI. } \\
\text { 14. I. } \\
\text { 8. III. } \\
\text { 16. IV. } \\
\text { 15. VI. }\end{array}$ & $\begin{array}{l}6,4 \\
6,7 \\
6,9 \\
6,8 \\
6,8\end{array}$ & $\begin{array}{l}20 \times 20 \\
15 \times 15 \\
25 \times 30 \\
25 \times 30 \\
25 \times 32\end{array}$ & $\begin{array}{r}103 \\
87 \\
61 \\
30 \\
30\end{array}$ & $\begin{array}{l}(+W) \\
(+t) \\
(H+) \\
(t+) \\
(t+)\end{array}$ & $\begin{array}{l}8700 \\
8600 \\
7800 \\
7600 \\
7400\end{array}$ & $\begin{array}{l}27,5 \\
25,0 \\
22,5 \\
25,0 \\
31,0\end{array}$ & $\begin{array}{l}(-) \\
+ \\
(+) \\
(+t) \\
(+H)\end{array}$ \\
\hline 13 & $\begin{array}{l}\text { W.T. } \\
\hat{\theta} \\
28 \mathrm{j} .\end{array}$ & $\begin{array}{l}\text { Frühin- } \\
\text { filtrat }\end{array}$ & $\begin{array}{l}\text { 27. X. } \\
\text { 8. I. } \\
\text { 16. III. } \\
\text { 21. V. }\end{array}$ & $\begin{array}{l}7,0 \\
6,8 \\
6,9 \\
6,9\end{array}$ & $\begin{array}{l}20 \times 20 \\
20 \times 20 \\
17 \times 19 \\
22 \times 24\end{array}$ & $\begin{array}{l}18 \\
16 \\
14 \\
18\end{array}$ & $\begin{array}{l}(+t) \\
(+) \\
(-)\end{array}$ & $\begin{array}{l}4400 \\
6200 \\
6800 \\
7400\end{array}$ & $\begin{array}{l}37,5 \\
31,0 \\
34,0 \\
33,5\end{array}$ & $\begin{array}{l}(-) \\
(+H) \\
(+H) \\
(+W)\end{array}$ \\
\hline 14 & $\begin{array}{l}\text { Y.G. } \\
20 \mathrm{j} .\end{array}$ & $\begin{array}{l}\text { Tub. } \\
\text { pulm. } \\
\text { duplex }\end{array}$ & $\begin{array}{l}\text { 22. X. } \\
\text { 24. XII. } \\
\text { 2. VI. } \\
\text { 13. VIII. }\end{array}$ & $\begin{array}{l}6,5 \\
6,5 \\
6,7 \\
7,7\end{array}$ & $\begin{array}{l}20 \times 20 \\
28 \times 28 \\
15 \times 15 \\
18 \times 20\end{array}$ & $\begin{array}{l}18 \\
44 \\
42 \\
25\end{array}$ & $\begin{array}{l}(H+) \\
(+t) \\
(+t) \\
(H+)\end{array}$ & $\begin{array}{l}5700 \\
8800 \\
6800 \\
7100\end{array}$ & $\begin{array}{l}33,0 \\
26,0 \\
33,0 \\
31,0\end{array}$ & $\begin{array}{l}(+) \\
(+H) \\
(+) \\
(-)\end{array}$ \\
\hline 15 & $\begin{array}{c}\text { T.Y. } \\
\hat{\delta} \\
26 \mathrm{j} .\end{array}$ & $\begin{array}{l}\text { Tub. } \\
\text { pulm. } \\
\text { duplex }\end{array}$ & $\begin{array}{l}\text { 27. XI. } \\
\text { 12. I. } \\
\text { 18. II. } \\
\text { 11. V. }\end{array}$ & $\begin{array}{l}6,9 \\
7,0 \\
7,0 \\
6,8\end{array}$ & $\begin{array}{l}10 \times 18 \\
15 \times 20 \\
20 \times 20 \\
20 \times 20\end{array}$ & $\begin{array}{l}38 \\
37 \\
34 \\
19\end{array}$ & $\begin{array}{l}(-) \\
(+) \\
(+) \\
(+1+)\end{array}$ & $\begin{array}{r}10400 \\
10000 \\
9900 \\
6150\end{array}$ & $\begin{array}{l}34,0 \\
32,0 \\
29,0 \\
25,0\end{array}$ & $\begin{array}{l}(+t) \\
(t+) \\
(+) \\
(-)\end{array}$ \\
\hline 16 & $\begin{array}{c}\text { W.P. } \\
\hat{8} \\
21 \mathrm{j} .\end{array}$ & $\begin{array}{l}\text { Tub. } \\
\text { pulm. } \\
\text { duplex }\end{array}$ & $\begin{array}{l}\text { 6. XI. } \\
\text { 8. I. } \\
\text { 7. IV. } \\
\text { 21. V. }\end{array}$ & $\begin{array}{l}7,3 \\
6,8 \\
7,0 \\
6,9\end{array}$ & $\begin{array}{l}20 \times 30 \\
30 \times 30 \\
20 \times 20 \\
23 \times 25\end{array}$ & $\begin{array}{l}90 \\
70 \\
19 \\
22\end{array}$ & $\begin{array}{l}(H+) \\
(+) \\
(+) \\
(+)\end{array}$ & $\begin{array}{r}11000 \\
7000 \\
8400 \\
6800\end{array}$ & $\begin{array}{l}21,0 \\
19,5 \\
28,0 \\
25,5\end{array}$ & $\begin{array}{l}(H+) \\
(+t) \\
(t+t)\end{array}$ \\
\hline 17 & $\begin{array}{c}\text { K.I. } \\
\hat{\delta} \\
21 \mathrm{j} .\end{array}$ & $\begin{array}{l}\text { Tub. } \\
\text { pulm. } \\
\text { duplex }\end{array}$ & $\begin{array}{l}\text { 3. XI. } \\
\text { 29. XII. } \\
\text { 18. II. } \\
\text { 7. IV. }\end{array}$ & $\begin{array}{l}6,6 \\
6,9 \\
6,7 \\
6,4\end{array}$ & $\begin{array}{l}20 \times 27 \\
19 \times 20 \\
35 \times 40 \\
25 \times 28\end{array}$ & $\begin{array}{l}50 \\
36 \\
38 \\
34\end{array}$ & $\begin{array}{l}(+) \\
(+) \\
(-)\end{array}$ & $\begin{array}{r}8600 \\
15700 \\
8000 \\
6600\end{array}$ & $\begin{array}{l}18,6 \\
19,5 \\
26,5 \\
25,0\end{array}$ & $\begin{array}{l}(+) \\
(+) \\
(++) \\
(+H)\end{array}$ \\
\hline 18 & $\begin{array}{c}\text { M.I. } \\
\hat{C} \\
26 \mathrm{j} .\end{array}$ & $\begin{array}{l}\text { Tub. } \\
\text { pulm. } \\
\text { duplex }\end{array}$ & $\begin{array}{l}\text { 3. XI. } \\
\text { 29. XII. } \\
\text { 12. I. } \\
\text { 8. III. }\end{array}$ & $\begin{array}{l}6,9 \\
6,5 \\
6,7 \\
6,8\end{array}$ & $\begin{array}{l}32 \times 32 \\
27 \times 33 \\
27 \times 30 \\
30 \times 30\end{array}$ & $\begin{array}{l}\mathbf{5 1} \\
36 \\
32 \\
17\end{array}$ & $\begin{array}{l}(+) \\
(t+) \\
(+t) \\
(-)\end{array}$ & $\begin{array}{r}8800 \\
10700 \\
8900 \\
8700\end{array}$ & $\begin{array}{l}23,5 \\
28,5 \\
18,5 \\
18,5\end{array}$ & $\begin{array}{l}(+) \\
(+) \\
(+H)\end{array}$ \\
\hline 19 & $\begin{array}{l}\text { S.T. } \\
\text { o } \\
23 . \mathrm{i} .\end{array}$ & $\begin{array}{l}\text { Tub. } \\
\text { pulm. } \\
\text { duplex }\end{array}$ & $\begin{array}{l}\text { 6. XI. } \\
\text { 8. I. } \\
\text { 16. III. } \\
\text { 31. V. }\end{array}$ & $\begin{array}{l}6,8 \\
6,7 \\
6,8 \\
6,8\end{array}$ & $\begin{array}{l}40 \times 40 \\
30 \times 30 \\
30 \times 34 \\
32 \times 35\end{array}$ & $\begin{array}{l}15 \\
43 \\
50 \\
25\end{array}$ & $\begin{array}{l}(-) \\
(-) \\
(-)\end{array}$ & $\begin{array}{l}8700 \\
8600 \\
6700 \\
5000\end{array}$ & $\begin{array}{l}54,0 \\
38,0 \\
51,0 \\
38,0\end{array}$ & $\begin{array}{l}(+H) \\
(-) \\
(H+1)\end{array}$ \\
\hline 20 & $\begin{array}{c}\text { K.M. } \\
\text { क } \\
16 \mathrm{j} .\end{array}$ & $\begin{array}{l}\text { Tub. } \\
\text { pulm. } \\
\text { duplex }\end{array}$ & $\begin{array}{l}\text { 12. I. } \\
\text { 16. III. } \\
\text { 15. VI. }\end{array}$ & $\begin{array}{l}6,7 \\
6,6 \\
6,5\end{array}$ & $\begin{array}{l}15 \times 18 \\
18 \times 20 \\
20 \times 94\end{array}$ & $\begin{array}{r}112 \\
91 \\
61\end{array}$ & $(-)$ & $\begin{array}{l}8900 \\
9700 \\
9300\end{array}$ & $\begin{array}{l}14,5 \\
14,0 \\
26,0\end{array}$ & $\begin{array}{l}(+t) \\
(+H) \\
(t+H)\end{array}$ \\
\hline
\end{tabular}


Tabelle II.

"Dynamische" Beobachtungen der entwicklunghemmenden Wirkung bei gestorbeve Fällen Lungentuberkulöser.

\begin{tabular}{|c|c|c|c|c|c|c|c|c|c|c|}
\hline 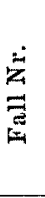 & 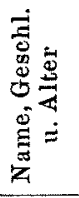 & Diagnose & Datum & 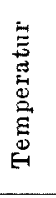 & 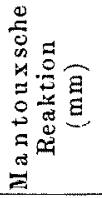 & 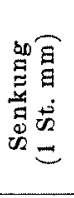 & 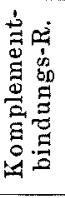 & 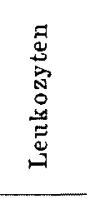 & 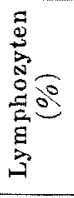 & 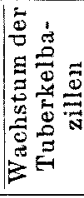 \\
\hline 1 & $\begin{array}{c}\text { H.K. } \\
\text { P } \\
22^{j} \mathrm{j} .\end{array}$ & $\begin{array}{l}\text { Tub. } \\
\text { pulm. } \\
\text { duplex }\end{array}$ & $\begin{array}{l}\text { 27. XI. } \\
12 . \mathrm{I} . \\
19 . \mathrm{V} . \\
\text { 9. VI. } \\
13 . \text { VIII. } \\
11 . \text { XI. } \\
15 . \mathrm{II} . \\
20 . \mathrm{IV} .\end{array}$ & $\begin{array}{l}7,0 \\
7,1 \\
7,0 \\
6,8 \\
7,0 \\
7,2 \\
8,3 \\
7,0\end{array}$ & $\begin{array}{c}31 \times 33 \\
20 \times 21 \\
35 \times 35 \\
23 \times 25 \\
30 \times 30 \\
20 \times 25 \\
25 \times 27 \\
0\end{array}$ & $\begin{array}{r}103 \\
95 \\
56 \\
85 \\
58 \\
100 \\
120 \\
102\end{array}$ & $\begin{array}{l}(+) \\
(+) \\
(++) \\
(+H) \\
(+H) \\
(+H) \\
(+) \\
(-)\end{array}$ & $\begin{array}{r}8700 \\
6300 \\
8600 \\
10800 \\
9800 \\
10200 \\
19400 \\
6700\end{array}$ & $\begin{array}{l}28 \\
26 \\
22,5 \\
17 \\
31,5 \\
30 \\
21 \\
24,5\end{array}$ & $\begin{array}{l}(H+) \\
(+) \\
(H+) \\
(+) \\
(-) \\
(+) \\
(H H)\end{array}$ \\
\hline 2 & $\begin{array}{c}\text { W.K. } \\
3 \\
15 \mathrm{j} .\end{array}$ & $\begin{array}{l}\text { Tub. } \\
\text { pulm. } \\
\text { duplex }\end{array}$ & $\begin{array}{l}\text { 27. XI. } \\
\text { 12. I. } \\
\text { 8. III. } \\
\text { 7. VI. } \\
\text { 18. II. }\end{array}$ & $\begin{array}{l}6,8 \\
6,4 \\
6,5 \\
6,8 \\
6,8\end{array}$ & $\begin{array}{l}30 \times 30 \\
25 \times 25 \\
20 \times 20 \\
23 \times 28 \\
20 \times 24\end{array}$ & $\begin{array}{l}33 \\
42 \\
23 \\
56 \\
42\end{array}$ & $\begin{array}{l}(-) \\
(++) \\
(+) \\
(+) \\
(-)\end{array}$ & $\begin{array}{r}6600 \\
11000 \\
10000 \\
5970 \\
8650\end{array}$ & $\begin{array}{l}26,5 \\
31,0 \\
29,0 \\
19,5 \\
24,0\end{array}$ & $\begin{array}{l}(+H) \\
(+) \\
(+t) \\
(H+) \\
(H+)\end{array}$ \\
\hline 3 & $\begin{array}{c}\text { W.S. } \\
\delta \\
18 \mathrm{j} .\end{array}$ & $\begin{array}{l}\text { Tub. } \\
\text { pulm. } \\
\text { duplex }\end{array}$ & $\begin{array}{l}\text { 3. XI. } \\
29 . \text { XII. } \\
\text { 16. III. } \\
10 . \mathrm{V} .\end{array}$ & $\begin{array}{l}7,3 \\
7,4 \\
7,6 \\
8,0\end{array}$ & $\begin{array}{c}13 \times 22 \\
17 \times 24 \\
23 \times 25 \\
0\end{array}$ & $\begin{array}{r}104 \\
107 \\
98 \\
41\end{array}$ & $\begin{array}{l}(t+) \\
(+) \\
(+t) \\
(-)\end{array}$ & $\begin{array}{r}10500 \\
9200 \\
11900 \\
13200\end{array}$ & $\begin{array}{r}16,0 \\
17,0 \\
9,0 \\
9,5\end{array}$ & $\begin{array}{l}(+) \\
(+) \\
(+) \\
(+t+1)\end{array}$ \\
\hline 4 & $\begin{array}{c}\text { S.M. } \\
\hat{\delta} \\
33 \mathrm{j} .\end{array}$ & $\begin{array}{c}\text { Tub. } \\
\text { pulm. } \\
\text { duplex }\end{array}$ & $\begin{array}{l}\text { 3. XI. } \\
\text { 29. XII. } \\
\text { 8. III. } \\
\text { 16. IV. } \\
\text { 15. VI. }\end{array}$ & $\begin{array}{l}7,5 \\
7,8 \\
8,0 \\
8,5 \\
8,0\end{array}$ & $\begin{array}{l}20 \times 22 \\
18 \times 20 \\
20 \times 25 \\
25 \times 25 \\
10 \times 10\end{array}$ & $\begin{array}{r}55 \\
86 \\
139 \\
135 \\
130\end{array}$ & $\begin{array}{l}(+) \\
(+) \\
(-) \\
(+) \\
(+)\end{array}$ & $\begin{array}{l}11000 \\
11300 \\
16600 \\
15200 \\
19200\end{array}$ & $\begin{array}{l}18,5 \\
20,5 \\
14,5 \\
13,0 \\
11,0\end{array}$ & $\begin{array}{l}(-) \\
(-) \\
(-) \\
(+) \\
(\text { HI) }\end{array}$ \\
\hline 5 & $\begin{array}{c}\text { S.Y. } \\
\hat{\jmath} \\
65 \mathrm{j}\end{array}$ & $\begin{array}{l}\text { Tub. } \\
\text { pulm. } \\
\text { duplex }\end{array}$ & $\begin{array}{l}\text { 3. XI. } \\
\text { 29. XII. } \\
\text { 7. IV. } \\
\text { 15. VI. } \\
\text { 13. IX. }\end{array}$ & $\begin{array}{l}6,6 \\
6,6 \\
6,6 \\
6,8 \\
6,6\end{array}$ & $\begin{array}{l}24 \times 26 \\
29 \times 30 \\
26 \times 28 \\
25 \times 25 \\
15 \times 15\end{array}$ & $\begin{array}{r}100 \\
90 \\
102 \\
91 \\
86\end{array}$ & $\begin{array}{l}(H+) \\
(+) \\
(-H) \\
(H A)\end{array}$ & $\begin{array}{r}8850 \\
8500 \\
11000 \\
6200 \\
13000\end{array}$ & $\begin{array}{l}30,5 \\
27,5 \\
27,0 \\
37,0 \\
30,0\end{array}$ & $\begin{array}{l}(t+) \\
(H+H) \\
(H+1) \\
(H+H) \\
(H+)\end{array}$ \\
\hline 6 & $\begin{array}{c}\text { H.T. } \\
\hat{\delta} \\
25 . j .\end{array}$ & $\begin{array}{l}\text { Tub. } \\
\text { pulm. } \\
\text { duplex }\end{array}$ & $\begin{array}{l}\text { 21. V. } \\
\text { 13. VIII. } \\
\text { 11. XI. } \\
\text { 17. I. }\end{array}$ & $\begin{array}{l}7,0 \\
7,3 \\
7,0 \\
7,0\end{array}$ & $\begin{array}{l}18 \times 22 \\
26 \times 30 \\
20 \times 20 \\
18 \times 26\end{array}$ & $\begin{array}{l}100 \\
102 \\
102 \\
107\end{array}$ & $\begin{array}{l}(\mathrm{HH}) \\
(\mathrm{HH}) \\
(\mathrm{H}) \\
(\mathrm{tt})\end{array}$ & $\begin{array}{r}12100 \\
10500 \\
10200 \\
9400\end{array}$ & $\begin{array}{l}31,0 \\
21,0 \\
27,5 \\
22,0\end{array}$ & $\begin{array}{l}(-) \\
(-) \\
(1+1)\end{array}$ \\
\hline 7 & $\begin{array}{c}\text { N.K. } \\
\delta \\
13 . j .\end{array}$ & $\begin{array}{c}\text { Pleuro- } \\
\text { peritonitis }\end{array}$ & $\begin{array}{l}\text { 8. IIT. } \\
\text { 16. IV. } \\
\text { 21. V. }\end{array}$ & $\begin{array}{l}6,8 \\
6,8 \\
8,0\end{array}$ & $\begin{array}{c}20 \times 20 \\
5 \times 6 \\
0\end{array}$ & $\begin{array}{r}100 \\
47 \\
20\end{array}$ & $\begin{array}{l}(-) \\
(-)\end{array}$ & $\begin{array}{r}10000 \\
8250 \\
7100\end{array}$ & $\begin{array}{l}22,0 \\
21,0 \\
18,0\end{array}$ & $\begin{array}{l}(\mathrm{HH}) \\
\mathrm{HH} \\
(\mathrm{HH})\end{array}$ \\
\hline 8 & $\begin{array}{c}\text { I.H. } \\
\text { 우 } \\
43^{\mathrm{j}} \mathrm{j} .\end{array}$ & $\begin{array}{l}\text { Tub. } \\
\text { pulm. } \\
\text { duplex }\end{array}$ & $\begin{array}{l}\text { 30. X. } \\
\text { 24. XII. } \\
\text { 16. III. }\end{array}$ & $\begin{array}{l}6,5 \\
7,6 \\
7,0\end{array}$ & $\begin{array}{r}25 \times 25 \\
20 \times 20 \\
7 \times 8\end{array}$ & $\begin{array}{l}100 \\
137 \\
108\end{array}$ & $\begin{array}{l}(++) \\
(+) \\
(-)\end{array}$ & $\begin{array}{l}4700 \\
5100 \\
4200\end{array}$ & $\begin{array}{l}27,5 \\
34,5 \\
39,0\end{array}$ & $\begin{array}{l}(-) \\
(+) \\
(++)\end{array}$ \\
\hline
\end{tabular}


der Bazillenentwicklung im Vollblut und Tuberkulosestadium, Körpertemperatur, Senkungsreaktion der Blutkörperchen, Komplementbindungsreaktion, Leukozyten- und Lymphozytenzahl Tuberkulöser sind aus den Tabellen III-IX zu ersehen. Auf die ,dynamische" Beobachtung dieser Verlältnisse legten wir das Hauptgewicht. Diese Ergebnisse sind in Tabelle I und II übersichtlich dargestellt. Auf diese beiden Tabellen werden wir in jedem Abschnitt einzugehen haben.

1. Beziehungen zwischen der entwicklunghemmenden

Wirkung des Vollbluts auf Tuberkelbazillen und dem Krankheitsstadium.

Teilt man die nach Aufnahme in die Klinik längere Zeit behandelten gebesserten und gestorbenen Fälle in 3 Gruppen ein, nämlich Gruppe I: Primärinfektion, Pleuritis und Peritonitis, Gruppe II : gebesserte Frühinfiltrate und Lungentuberkulose und Gruppe III: gestorbene Lungentuberkulose, und vergleicht man die Versuchsergebnisse diser 3 Gruppen miteinander, so kann man daraus ersehen, wie sich die entwicklunghemmende Wirkung des Vollbluts auf die Tuherkelbazillen je nach dem Krankheitsstadium zeigte.

Nach den Ergebnissen von Ima mura und Schibuk a w a beträgt der Prozentsatz des Bazillen wachstums beiGesunden $100 \%$, bei Tuberkuloseverdächtigen $82,9 \%$ bei Frühinfiltraten $8,6 \%$, bei leichter Lungentuberkulose $15,9 \%$, bei mittelschwerer $0 \%$ und bei schwerer Tuberkulose $100 \%$.

Unsere Ergebnisse in Tabelle III zeigen, dass diese Prozentsätze

$$
\text { Tabelle III. }
$$

Entwicklunghemmende Wirkung und Krankheitsstadien.

\begin{tabular}{|c|c|c|c|c|}
\hline \multirow[b]{2}{*}{$\begin{array}{l}\text { Bazillen- } \\
\text { wachstum }\end{array}$} & \multicolumn{3}{|c|}{ Gruppe } & \multirow[b]{2}{*}{ Gesamtfälle } \\
\hline & $\begin{array}{c}\text { I } \\
\text { Primärinfektion, } \\
\text { Pleuritis und } \\
\text { Peritonitis }\end{array}$ & $\begin{array}{c}\text { II } \\
\text { Frühinfiltrat } \\
\text { und } \\
\text { Tuberculosis } \\
\text { pulmonum } \\
\text { (gebessert) }\end{array}$ & $\begin{array}{c}\text { III } \\
\text { Tuberculosis } \\
\text { pulınonum } \\
\text { (gestorben) }\end{array}$ & \\
\hline $\begin{array}{c}(\text { HW }) \\
(H) \\
(++) \\
(+) \\
(-) \\
\text { Gesamtzahl }\end{array}$ & $\begin{array}{c}12 \\
20 \\
11\}(40,4 \%) \\
8\} \\
21 \\
1\}(17,3 \%) \\
\begin{array}{c}52 \\
5\end{array} \\
(40)\end{array}$ & $\begin{array}{l}22 \quad(9,7 \%) \\
53\} 108 \\
55\}(47,5 \%) \\
55\} \quad 97 \\
42\}(42,7 \%) \\
\quad 227\end{array}$ & $\begin{array}{c}10(17,2 \%) \\
13) \quad 20 \\
7\}(34,5 \%) \\
12) 28 \\
16)(48,2 \%) \\
58\end{array}$ & $\begin{array}{l}44(13,1 \%) \\
86\} 159 \\
73\}(47,1 \%) \\
75\} 134 \\
59\}(39,7 \%) \\
\quad 337\end{array}$ \\
\hline
\end{tabular}


bei Gruppe I $23 \%$, bei Gruppe II 9,7\% und bei Gruppe III 17,2\% betragen.

Zwischen dem Ergebnis beider Autoren und dem unsrigen besteht ein bedeutender Uuterschied. Das kommt wohl daher, dass die Einteilung der Stadien, die Zeit der Kultivierung, die Bestimmung des Bazillenwachstums u.a.m. verschieden sind. Vor allem ist die Wachstumsfähigkeit des benutzten Bazillenstamms zu berücksichtigen. Dass sich der Prozentsatz ihres Wachstums trotzdem mit den weiteren Stadien der Tuberkulose allmählich vermindert, dagegen bei den sterbenden Fällen wieder steigt, darin herrscht Übereinstimmung.

Bei unserem Versuche gibt es auch bei den gebesserten Fällen von Frühinfiltrat und Lungentuberkulose Fälle, bei welchen das Bazillenwachstum ebenso lebhaft wie bei den gestorbenen Fällen ist: Nr. 8, 12, 13, 17 in Tabelle I und II. Dies kann darauf beruhen, dass sich die sterbenden Fälle der negativen Anergie genähert haben, die gebesserten Fälle hingegen positiv anergetisch geworden sind.

2. Beziehungen zwischen der entwicklunghemmenden Wirkung des Vollbluts und der Körpertemperatur.

Temperatursteigerung ist eine Tuberkulösen eigentümliche Erscheinung. Die Ursache liegt in der unmittelbaren Reizung des Wärmezentrums und der Funktionstörung der Wärmekontrolle durch das Bazillengift, das andererseits die Lungengefässe erweitert und die peripheren Blutgefässe verengert. Im allgemeinen beginnt die klinisch zu diagnostizierende Primärinfektion mit hohem Fieber, bekommt danach gewöhnlich remittierendes Fieber, aber auch beim Frühinfiltrat sowie bei der Lungenphthise ist die Körpertemperatur wechselnd, bald normal, bald beim Schub Fieber verschiedener Typen, nur bei schwerer Tuberkulose oft hohes Fieber, beinahe wie febris hectica; bei Er'weiterung und Einschmelzen der infiltierten Herde können febris continua oder febris typo inverso usw. auftreten. Deshalb ist es unmöglich, nur aus den Fiebertypen das Stadium der Tuberkulose zu kennen.

Nach Tabelle IV zeigt Gruppe I meistens eine Körpertemperatur von etwa 36,9 bis $37,5^{\circ} \mathrm{C}$, Gruppe II gewölnnlich normale Körpertemperatur, aber Gruppe III mehr Fälle mit Körpertemperatur über $37,6^{\circ} \mathrm{C}$ als Gruppe II. Das zeigt, dass sich die Fälle hoher Temperatursteigerung mit den weitergehenden Tuberkulosestadien vermehren. Bei der Zusammenfassung aller Fälle betragen die mit normaler Kör- 
Tabelle IV.

Entwicklunghemmende Wirkung und Körpertemperatur.

\begin{tabular}{|c|c|c|c|c|}
\hline \multirow{3}{*}{$\begin{array}{l}\text { Bazillen- } \\
\text { wachstum }\end{array}$} & \multicolumn{4}{|c|}{$\begin{array}{c}\text { Gruppe I } \\
\text { Primärinfektion, Pleuritis und Peritonitis }\end{array}$} \\
\hline & \multicolumn{4}{|c|}{ Körpertemperatur } \\
\hline & $36,8^{\circ} \mathrm{C}$ & $\begin{array}{l}36,9- \\
37,5^{\circ} \mathrm{C}\end{array}$ & $37,6-$ & $\begin{array}{l}38,1^{\circ} \mathrm{C} \\
+\end{array}$ \\
\hline $\begin{array}{c}(\text { HI) } \\
(+H) \\
(+t) \\
(+) \\
(-) \\
\text { Gesamtzahl }\end{array}$ & $\begin{array}{lc}6 & (26 \%) \\
6\} & 12 \\
6\} & (53 \%) \\
4 & 5 \\
1 & 5 \\
(21,3 \%) \\
23\end{array}$ & $\begin{array}{c}6 \\
12)(23 \%) \\
4\}(61,5 \%) \\
4 \\
0\}(15,6 \%) \\
4 \\
26\end{array}$ & $\begin{array}{c}0 \\
3 \\
1\}(100 \%) \\
0 \\
3\end{array}$ & $\begin{array}{l}0 \\
0 \\
0 \\
0\end{array}$ \\
\hline \multirow{3}{*}{$\begin{array}{l}\text { Bazillen- } \\
\text { wachstum }\end{array}$} & \multicolumn{4}{|c|}{$\begin{array}{l}\text { Gruppe II } \\
\text { berculosis pulmonnm (gebessert) }\end{array}$} \\
\hline & \multicolumn{4}{|c|}{ Körpertemperatur } \\
\hline & $\overline{36,8^{\circ} \mathrm{C}}$ & $\begin{array}{l}36,9- \\
37,5^{\circ} \mathrm{C}\end{array}$ & $\begin{array}{l}37,6- \\
38,0^{\circ} \mathrm{C}\end{array}$ & $\begin{array}{c}38,1^{\circ} \mathrm{C} \\
+\end{array}$ \\
\hline 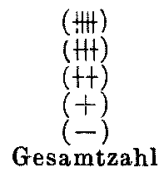 & $\begin{array}{l}11 \quad(8,7 \%) \\
30\} 61 \\
31\}(48,3 \%) \\
\left.30\} \begin{array}{r}54 \\
24\end{array}\right\}\left(\begin{array}{c}42,8 \%) \\
126\end{array}\right.\end{array}$ & $\begin{array}{l}11(12,6 \%) \\
20\{61 \\
21\}(47,1 \%) \\
21\{35 \\
14\{(40,2 \%) \\
87\end{array}$ & 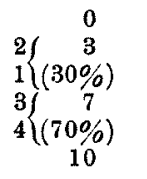 & $\begin{array}{l}1 \\
1 \\
2\} \\
1 \\
(75 \%) \\
0 \\
0 \\
(25 \%) \\
4\end{array}$ \\
\hline \multirow{3}{*}{$\begin{array}{l}\text { Bazillen- } \\
\text { wachstum }\end{array}$} & \multicolumn{4}{|c|}{$\begin{array}{c}\text { Gruppe II } \\
\text { Tuberculosis pulmonum (gestorben) }\end{array}$} \\
\hline & \multicolumn{4}{|c|}{ Körpertemperatur } \\
\hline & $36, \overline{8}^{\circ} \mathrm{C}$ & $\begin{array}{l}36,9- \\
37,5^{\circ} \mathrm{C}\end{array}$ & $\begin{array}{l}37,6- \\
38,0^{\circ} \mathrm{C}\end{array}$ & $\begin{array}{c}38,1^{\circ} \mathrm{C} \\
+\end{array}$ \\
\hline $\begin{array}{c}(\text { HH) } \\
(H) \\
(++) \\
(+) \\
(-) \\
\text { Gesamtzahl }\end{array}$ & 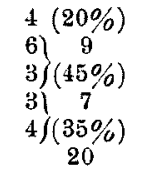 & 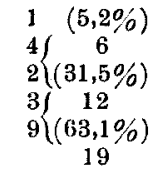 & 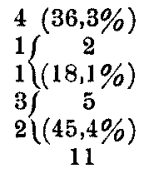 & 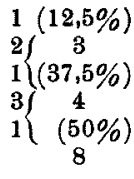 \\
\hline \multirow{3}{*}{$\begin{array}{l}\text { Bazillen- } \\
\text { wachstum }\end{array}$} & \multicolumn{4}{|c|}{ Gesamtfälle } \\
\hline & \multicolumn{4}{|c|}{ Körpertemperatur } \\
\hline & $36, \overline{8}^{\circ} \mathrm{C}$ & $\begin{array}{l}36,9- \\
37,5^{\circ} \mathrm{C}\end{array}$ & $\begin{array}{l}37,6- \\
38,0^{\circ} \mathrm{C}\end{array}$ & $\begin{array}{c}38,1^{\circ} \mathrm{C} \\
38,1^{\circ} \mathrm{C} \\
+\end{array}$ \\
\hline $\begin{array}{c}(H+) \\
(H+) \\
(+t) \\
(+) \\
(-) \\
\text { Gesamtzahl }\end{array}$ & 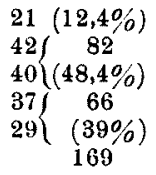 & 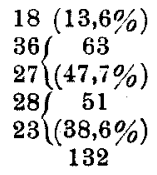 & 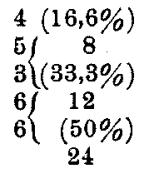 & 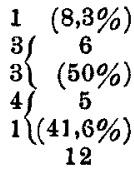 \\
\hline
\end{tabular}


pertemperatur $169(50,7 \%)$, die mit $36,9-37,5^{\circ} \mathrm{C} 132(39,2 \%)$, die mit $37,6-38,0^{\circ} \mathrm{C} 24(7,1 \%)$ und die über $38,1^{\circ} \mathrm{C} 12(3,5 \%)$. Also sind die Fälle mit normaler Körpertemperatur in der Klinik am häufigsten vertreten. Wright hatte zwar mitgeteilt, dass im Blut fiebernder tuberkulöser Individuen das Wachstum der Tuberkelbazillen gehemmt wird. Wenn man aber unsere Fälle betrachtet, so bemerkt man, dass in Gruppe I sich der Wachstumsgrad der Bazillen gleichmässig mit der Temperaturzunahme vermehrt. Anderseits zeigt bei Gruppe II das Bazillenwachstum (H) bei normaler Temperatur und das $(+)$ und $(-)$ bei $37,6-38,0^{\circ} \mathrm{C}$ den höchsten Wert. Bei Gruppe III liegt der höchste Wert des Bazillenwachstums (卅) bei $37,6-38,0^{\circ} \mathrm{C}$ und der des $(+)$ und $(-)$ bei $36,9-37,5^{\circ} \mathrm{C}$.

Zusammengefasst, nimmt das Bazillenwachstum(Hit) mit der Temperatursteigerung in dieser Reihenfolge zu : $12,4 \%, 13,6 \%$ und $16,6 \%$, hingegen bei über $38,1^{\circ} \mathrm{C}$ umgekehrt bis auf $8,3 \%$ ab. Das Bazillenwachstum (+) und (-) nimmt in dieser Reihenfolge zu : 39\%, 38,6\% und $50 \%$, bei über $38,1^{\circ} \mathrm{C}$ jedoch sinkt es wieder bis auf $41,6 \%$. „Dynamisch" betrachtet, ist aber die Beziehung des Bazillenwachstums zur Temperaturschwankung, wie bei Nr. 2, 14, 16 in Tabelle I und Nr. 1 und 3 in Tabelle II ersichtlich, nicht auffallend.

3. Beziehungen zwischen der entwicklunghemmenden

Wirkung des Vollbluts auf Tuberkelbazillen und der Tuberkulinallergie.

Wir verwendeten zur Prüfung der Tuberkulinüberempfindlichkeit der Haut die Mendel-Man to uxsche Reaktion und untersuchten sie mit 0,1 ccm einer 1:1000 verdünnten Alttuberkulinlösung. Die Rötung unter $4 \mathrm{~mm}$ wird als negativ angesehen. Die positiven Ausfälle wurden je nach der Ausdehung der Rötung in 4 Grade eingeteilt: 5-15 $\mathrm{mm}(+), 16-25 \mathrm{~mm}(++), 26-35(\mathrm{Ht})$ und über $36(\mathrm{HH})$.

Die Zeit, innerhalb derer nach Tuberkuloseinfektion Tuberkulinallergie eintritt, wird Inkubationszeit genannt; dabei ist die Senkungsgeschwindigkeit der Erythrozyten vielfach beschleunigt im Vergleich zu anderen Symptomen; zuweilen lässt sich dabei auch ein Schatten von der Primärinfiltrierung im Röntgenbild der Lunge erkennen.

Bei Tuberkuloseinfektion nimmt die Tuberkulinallergie mit fortschreitender Krankheit immer mehr zu; bei Besserung sowie Verschlimmerung nimmt sie wieder ab, aber mehrere Jahre sollen erfor- 


\section{Tabelle V.}

Entwicklunghemmende Wirkung und Tuberkulinallergie.

\begin{tabular}{|c|c|c|c|c|c|}
\hline \multirow{3}{*}{$\begin{array}{l}\text { Bazillen- } \\
\text { wachstum }\end{array}$} & \multicolumn{5}{|c|}{$\begin{array}{c}\text { Gruppe I } \\
\text { Primärinfektion, Pleuritis und Peritonitis }\end{array}$} \\
\hline & \multicolumn{5}{|c|}{ Mantouxsche Reaktion } \\
\hline & $0-5 \mathrm{~mm}$ & $6-15 \mathrm{~mm}$ & $16-25 \mathrm{~mm}$ & $26 \sim 35 \mathrm{~mm}$ & $36+\mathrm{mm}$ \\
\hline $\begin{array}{c}(\mathrm{HH}) \\
(\mathrm{HH}) \\
(\mathrm{tt}) \\
(+) \\
(-) \\
\text { Gesamtzahl }\end{array}$ & 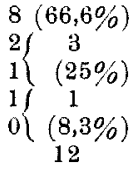 & 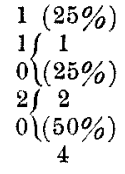 & 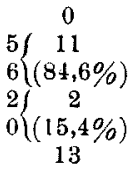 & 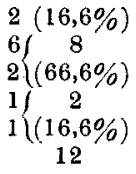 & 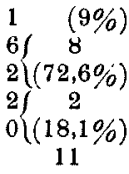 \\
\hline
\end{tabular}

Gruppe II

Bazillen-

Frühinfiltrat und Tuberculosis pulmonnm (gebessert)

\begin{tabular}{|c|c|c|c|c|c|}
\hline \multirow[t]{2}{*}{ wachstum } & \multicolumn{5}{|c|}{ Mantouxsche Reaktion } \\
\hline & $0-5 \mathrm{~mm}$ & $6-15 \mathrm{~mm}$ & $16-25 \mathrm{~mm}$ & $26-35 \mathrm{~mm}$ & $36+\mathrm{mm}$ \\
\hline $\begin{array}{c}(\text { tit) } \\
(\mathrm{tt}) \\
(t+) \\
(+) \\
(-) \\
\text { Gesamtzahl }\end{array}$ & $\begin{array}{l}1(50 \%) \\
1) 1 \\
0(50 \%) \\
0 \\
2\end{array}$ & 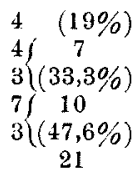 & 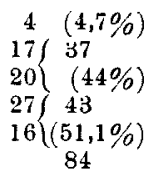 & $\begin{array}{c}10(12,5 \%) \\
13\{30 \\
20((41,2 \%) \\
16\} 37 \\
21((46,2 \%) \\
80\end{array}$ & $\begin{array}{c}3(6 \%) \\
18(40 \\
12\{(80 \%) \\
5\{7 \\
2\{(14 \%) \\
50\end{array}$ \\
\hline \multirow{3}{*}{$\begin{array}{l}\text { Bazillen- } \\
\text { wachstum }\end{array}$} & \multicolumn{5}{|c|}{$\begin{array}{c}\text { Gruppe III } \\
\text { Tuberculosis pulmonum (gestorben) }\end{array}$} \\
\hline & \multicolumn{5}{|c|}{ Mantouxsche Reaktion } \\
\hline & $0-5 \mathrm{~mm}$ & $6-15 \mathrm{~mm}$ & $16-25 \mathrm{~mm}$ & $26-35 \mathrm{~mm}$ & $36+\mathrm{mm}$ \\
\hline $\begin{array}{c}(\text { tHt }) \\
(\mathrm{Ht}) \\
(+t) \\
(+) \\
(-) \\
\text { Gesamtzahl }\end{array}$ & 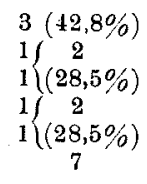 & $\begin{array}{l}3(37,5 \%) \\
0 f \quad 2 \\
2)(25 \%) \\
0\} \quad 3 \\
3\left(\begin{array}{c}(37,5 \%) \\
8\end{array}\right.\end{array}$ & $\begin{array}{l}2(6,6 \%) \\
7\{11 \\
4\{(36,6 \%) \\
9\{17 \\
8\{(56,6 \%) \\
30\end{array}$ & $\begin{array}{l}2(16,6 \%) \\
4\left\{\begin{array}{c}4 \\
0(33,3 \%)\end{array}\right. \\
2\left\{\begin{array}{c}6 \\
4(50 \%) \\
12\end{array}\right.\end{array}$ & 1) $\begin{array}{c}0 \\
1 \\
(100 \%) \\
0 \\
1\end{array}$ \\
\hline \multirow{3}{*}{$\begin{array}{l}\text { Bazillen- } \\
\text { wachstum }\end{array}$} & \multicolumn{5}{|c|}{ Gesamtfälle } \\
\hline & \multicolumn{5}{|c|}{ Mantoux sche Reaktion } \\
\hline & $0-5 \mathrm{~mm}$ & $6-15 \mathrm{~mm}$ & $16-25 \mathrm{~mm}$ & $26-35 \mathrm{~mm}$ & $36+\mathrm{mm}$ \\
\hline $\begin{array}{c}(+H) \\
(H+) \\
(+t) \\
(+) \\
(-) \\
\text { Gesaimtzahl }\end{array}$ & $\begin{array}{c}12(57,1 \%) \\
4 f 6 \\
2)(28,5 \%) \\
2 f 3 \\
1((14,3 \%) \\
21\end{array}$ & $\begin{array}{l}8(24,2 \%) \\
5\{10 \\
5\{(30,3 \%) \\
9\} 15 \\
6\left(\begin{array}{c}(45,4 \%) \\
33\end{array}\right.\end{array}$ & $\begin{array}{cc}6 & (4,6 \%) \\
29 & 59 \\
30 & (45,7 \%) \\
38 & 62 \\
24 & (48 \%) \\
129 & \end{array}$ & $\begin{array}{l}14(13,7 \%) \\
23(45 \\
22\{(44,1 \%) \\
19\{45 \\
26\{(44,1 \%) \\
102\end{array}$ & 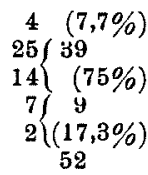 \\
\hline
\end{tabular}


derlich sein, bis die Tuberkulinallergie bei der Besserung abnimmt, und nur äusserst selten verschwindet sie völlig.

Tabelle V zeigt, dass in Gruppe I die mit den Tuberkulinreaktionen $(-),(t+)$ und $(H t)$ am zahlreichsten sind, aber in Gruppe II die mit $(+t),(H)$ und $(H+H)$, dagegen die mit $(-)$ beschränkt sich nur auf 2 Fälle $(0,8 \%)$; in Gruppe III gibt es jedoch die mit $(+t)$ am meisten, dann die mit (Ht) nnd (+), unil die mit (-) sind zu 12,8 und $7(12 \%)$ vertreten. Dies zeigt, dass das Wachstum der Tuberkelbazillen umgekehrt zur Tuberkulinallergie verläuft und bei denen mit negativer Anergie ebenso lebhaft wie bei Gesunden ist. Aus Tabelle V kann man ferner erkennen, dass bei Gruppe I das Bazillenwachstum (卅) mit fortschreitender Tuberkulinallergie allmählich so abnimmt: $66,6 \%$, $25 \%, 16,6 \%$ und $9 \%$, das Bazillenwachstum $(+)^{\circ}$ und $(-)$ dagegen so zunimmt : 8,3\%, 15,4\%, 16,6\% und 18,1\%. Bei den Gruppen II und III vermehren sich die, welche bei positiver Tuberkulinallergie das Bazillenwachstum (冊) zeigen, und vermindern sich die Fälle, welche $(+)$ und $(-)$ zeigen. Dies kommt daher, dass trotz vorläufiger $\mathrm{Zu}-$ nahme oder Unverändertbleiben der Tuberkulinallergie bei Gruppe II (Fälle Nr. 8, 12 und 17 in Tabelle I) das Bazillenwachstum immer besser. wird, und dass bei Gruppe III (Fälle Nr. 1, 3, 7 und 8 in Tabelle II) das Bazilenwachstum immer stärker wird, die Tuberkulinallergie hingegen allmählich abnimmt und sich der negativen Anergie nähert. Aber derGrad vonTuberkulinallergie nimmt bei ein und demselbenIndividuum stets ab oder zu, und dass das Bazillenwachstum dadurch nicht beeinflusst wird, ist aus Tabelle I und II ersichtlich. Man kann nur sagen, dass das Bazillenwachstum im grossen und ganzen dazu neigt, entgegengesetzt zur Tuberkulinallergie zu verlaufen. Dies kommt daher, dass zwischen der entwicklunghemmenden Wirkung auf Tuberkelbazillen, die die humorale Allergie ausmacht, und der Tuberkulinallergie, die für einen Teil der Gewebsallergie zu halten ist, immunologisch ein natürlicher Unterschied besteht; auch von diesem Standpunkt aus lässt sich die Ansicht von Alva re y u. Barralt, ${ }^{22)}$ der Immunitätszustand der Tuberkulose könne nicht durch die Tuberkulinallergie festgestellt werden, bestätigen. Was die zeitliche Beziehung zwischen dem Auftreten der Tuberkulinallergie und dem der entwicklunghemmenden Wirkung des Vollbluts auf Tuberkelbazillen betrifft, so berichteten Ito, Ogat a und S c h ibuk a wa, dass beide Reaktionen im allgemeinen gleichzeitig auftreten. Nach unserer Untersuchung beträgt bei einem Teil der ersten Gruppe in Tabelle V, bei dem die Tuberkulinallergie negativ ist, das Bazillenwachst um bei 8 Fällen $(66,6 \%)$ (州) 
und das bei 4 Fällen $(33,3 \%)(+)$ und (-). Dies deutet darauf hin, dass bei $\frac{2}{3}$ der Fälle die entwicklunghemmende Wirkung nach Entstehung der Tuberkulinallergie auftritt. Später verläuft die entwicklunghemmende Wirkung im allgemeinen parallel mit der Tuberkulinallergie. Nur bei den sich bessernden Fällen, wie Nr. 8 und 12 in Tabelle I, wird das Wachstum schon lebhafter, wenn die Tuberkulinallergie noch nicht geschwächt ist, und bei Sterbenden, die in negative Anergie gerieten, wird es, z. B. bei Nr. 1 und 7 in Tabelle II, erst später lebhaft, wenn die Tuberkulinallergie allmählich abnimmt.

4. Beziehungen zwischen der entwicklunghernmenden Wirkung des Vollbluts auf Tuberkelbazillen und der Senkungsgeschwindigkeit der Blutkörperchen.

Die Senkungsreaktion der Blutkörperchen wird beschleunigt, wenn im Körper ein pathologischer Zerfall vor sich geht, also ist sie keine spezifische Reaktion der Tuberkulose. Aber die Tuberkuloseinfektion hat zweifellos pathologischen Gewebszerfall im Körper zur Folge, so dass die Blutkörperchensenkung beschleunigt wird. Deshalb ist diese nichtspezifische Reaktion diagnostisch sowie prognostisch eine unentbehrliche Hilfe bei der Klinik der Tuberkulose. Noch ehe sich bei der Primärinfektion der Tuberkulose, bevor noch irgendwelche subjektiven Klagen vorgebracht werden, positive Tuberkulinreaktion und röntgenologisch Veränderungen an der Brust feststellen lassen, ist die Blutsenkung schon oft beschleunigt, z. B. bei den Fällen Nr. 1, 2 und 3 in Tabelle 1. Falls die Senkungsgeschwindigkeit der roten Blutzellen in diesem Frühstadium hochgradig beschleunigt ist, entsteht häufig Pleuritis oder Pleuroperitonitis.

Von Westergren ${ }^{233}$ und a.m. ist bewiesen worden, dass die Beschleunigung der Blutsenkung im allgemeinen Verschlimmerung der Tuberkulose und ungekehrt ihre Verlangsammung Besserung der Krankheit bedeutet. Dass sie auch im schweren Stadium der Tuberkulose wieder abnimmt, ist von Westergren, Katz, ${ }^{24)} \mathrm{Katsunu}$ $\mathrm{ma},{ }^{25)} \mathrm{Okabe}^{(8)}$ u. a. ebenfalls anerkannt worden. Es ist bemerkenswert, dass sich diese Reaktion, wenn sie auch keine spezifische Reaktion darstellt, doch ganz ähnlich wie diese verändert, d. h. Tuberkulinallergie, Komplementbindungsreaktion usw.

Die Fälle Nr. 1, 2, 7 u. 9 in Tabelle I und die Fälle Nr. 2 u. 7 in Tabelle II zeigen, dass bei Tuberkuloseinfektion Beschleunigung der 


\section{Tabelle VI.}

Eutwicklunghemmende Wirkung und Senkungsgeschwindigkeit der roten Blutkörperchen.

\begin{tabular}{|c|c|c|c|c|c|c|}
\hline \multirow{3}{*}{$\begin{array}{l}\text { Bazillen- } \\
\text { wachstum }\end{array}$} & \multicolumn{6}{|c|}{$\begin{array}{c}\text { Gruppe I } \\
\text { Primärinfektion, Pleuritis und Peritonitis }\end{array}$} \\
\hline & \multicolumn{6}{|c|}{ Senkungsgeschwindigkeit } \\
\hline & $0-15$ & $16-35$ & $36-55$ & $56-75$ & $76-95$ & $96+$ \\
\hline $\begin{array}{c}(+H) \\
(H+) \\
(t+) \\
(+) \\
(-) \\
\text { Gesamtzahl }\end{array}$ & $\begin{array}{lc}5 & (33,3 \%) \\
6) & 9 \\
3 f & (60 \%) \\
1) \\
0) \\
(6,6 \%) \\
15\end{array}$ & 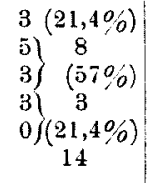 & $\begin{array}{l}2(15,3 \%) \\
4) \\
6 \\
2\} \\
4\} \\
4(46 \%) \\
1)(38,4 \%) \\
13\end{array}$ & $\begin{array}{c}2(33,3 \%) \\
2) \\
2)(66,6 \%) \\
0 \\
6\end{array}$ & $\begin{array}{l}0 \\
0 \\
0 \\
0\end{array}$ & $\begin{array}{c}0 \\
\text { 3) } \\
4 \\
(100 \%) \\
0 \\
4\end{array}$ \\
\hline \multirow{3}{*}{$\begin{array}{l}\text { Bazillen- } \\
\text { wachstum }\end{array}$} & \multicolumn{6}{|c|}{$\begin{array}{l}\text { Gruppe II } \\
\text { Frühinfiltrat und Tuberculosis pulmonum (gebessert) }\end{array}$} \\
\hline & \multicolumn{6}{|c|}{ Senkungsgeschwindigkeit } \\
\hline & $0-15$ & $16-35$ & $35-55$ & $56-75$ & $76-95$ & $96+$ \\
\hline $\begin{array}{c}(\text { (WI) } \\
(\mathrm{Ht}) \\
(++) \\
(+) \\
(-) \\
\text { Gesamtzahl }\end{array}$ & 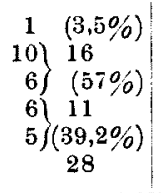 & $\begin{array}{l}10(13,3 \%) \\
16) 33 \\
17\}(44 \%) \\
18\} 32 \\
14)(42,6 \%) \\
75\end{array}$ & $\begin{array}{cl}5 & (10 \%) \\
9 & 22 \\
13\} & (14 \%) \\
13\} & 23 \\
10 & (46 \%) \\
& 50\end{array}$ & $\begin{array}{cc}2 & (6,4 \%) \\
5) & 15 \\
10 & (48,3 \%) \\
7 & 14 \\
7 & (45,1 \%) \\
31\end{array}$ & $\begin{array}{lc}2 & (8,7 \%) \\
9 & 14 \\
5\} & (65,1 \%) \\
5\} & 7 \\
2\} & (32,5 \%) \\
& 23\end{array}$ & $\begin{array}{ll}2 & (10 \%) \\
4) & 8 \\
4\} & (40 \%) \\
6) & 10 \\
4\} & (50 \%) \\
& 20\end{array}$ \\
\hline \multirow{3}{*}{$\begin{array}{l}\text { Bazillen- } \\
\text { waehstum }\end{array}$} & \multicolumn{6}{|c|}{$\begin{array}{l}\text { Gruppe III } \\
\text { Tuberculosis pulmonum (gestorben) }\end{array}$} \\
\hline & \multicolumn{6}{|c|}{ Senknngsgeschwindigkeit } \\
\hline & $0-15$ & $16-3 \overline{5}$ & $36-55$ & $56-75$ & $76-95$ & $96+$ \\
\hline $\begin{array}{c}(+ \text { H) } \\
(+t) \\
(++) \\
(+) \\
(-) \\
\text { Gesamtzahl }\end{array}$ & $\begin{array}{l}0 \\
0 \\
0 \\
0\end{array}$ & $\begin{array}{lc}1 & (25 \%) \\
1\} & 2 \\
1\} & (50 \%) \\
0\} & 1 \\
1\} & (25 \%) \\
4\end{array}$ & $\begin{array}{l}1(14,3 \%) \\
2) \quad 3 \\
1)(42,8 \%) \\
1) \quad 3 \\
2 \int(42,8 \%) \\
7\end{array}$ & $\begin{array}{l}0 \\
4\} \\
5 \\
1\}(55,5 \%) \\
1\} \begin{array}{c}4 \\
3\}(44,4 \%) \\
9\end{array}\end{array}$ & $\begin{array}{l}3(27,2 \%) \\
3) 4 \\
1\}(36,3 \%) \\
2) 4 \\
2\}(36,3 \%) \\
11\end{array}$ & $\begin{array}{l}5(18,8 \%) \\
3) \quad 6 \\
3\}(22,2 \%) \\
8\} 16 \\
8\}(59 \%) \\
27\end{array}$ \\
\hline \multirow{3}{*}{$\begin{array}{l}\text { Bazillen- } \\
\text { wachstum }\end{array}$} & \multicolumn{6}{|c|}{ Gesamtfälle } \\
\hline & \multicolumn{6}{|c|}{ Senkungsgeschwindigkeit } \\
\hline & $0-15$ & $16-35$ & $36-5 \overline{5}$ & $56-75$ & $76-95$ & $96+$ \\
\hline $\begin{array}{c}(\text { (HI) } \\
(+H) \\
(+t) \\
(+) \\
(-) \\
\text { Gesamtzahl }\end{array}$ & $\begin{array}{c}6(13,9 \%) \\
16) 25 \\
9)(58,1 \%) \\
7) 12 \\
5)(27,9 \%) \\
43\end{array}$ & $\begin{array}{cc}14 & (15 \%) \\
22) & 43 \\
21 & (46,2 \%) \\
21) & 36 \\
15\} & (38,7 \%) \\
& 93\end{array}$ & $\begin{array}{c}8(11,4 \%) \\
15)(31 \\
16\}(44,3 \%) \\
18\} 31 \\
13\}(44,3 \%) \\
70\end{array}$ & $\begin{array}{c}4(8,7 \%) \\
11) 24 \\
13)(52,2 \%) \\
8\} 18 \\
10)(39,1 \%) \\
46\end{array}$ & $\begin{array}{r}5(14,7 \%) \\
12) 18 \\
6\} \\
\left.7\} \begin{array}{l}(53 \%) \\
11 \\
4\end{array}\right\}(32,3 \%) \\
34\end{array}$ & 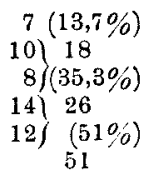 \\
\hline
\end{tabular}


Senkung der roten Blutzellen früher als Tuberkulinallergie und Komplementbindungsreaktion auftritt und auf dem Wege zur Heilung auch wieder zu früherer Abnahme neigt; aber sie scheint, wie Spies ${ }^{27}$ betonte, auch beim Endstadium der Tuberkulose, wo die Tuherkulinallergie und Komplementbindungsreaktion negative Anergie zeigen, eine Weile im Beschleunigungszustand zu verweilen und unmittelbar vor dem Tode plötzlich zum normalen Werte zurückzukehren.

Deshalb gibt es ziemlich viele Fälle, die sterben, bevor die Senkungsgeschwindkeit zu ihrem normalen Wert zurückgekehrt ist. Die Fälle Nr. 1, 3 u. 5 in Tabelle II stellen dafür passende Beispiele dar. Aus Tabelle VI ist zu ersehen, dass die Blutsenkung bei der Mehrzahl der Fälle von Gruppe I ihren normalen Wert zeigte, dass hingegen die Zahl der Fälle mit stark beschleunigter Blutsenkungsgeschwindigkeit allmählich abnimmt; bei 4 Fällen, welche sehr hochgradige Beschleunigung zeigten, lag Pleuritis vor.

Wenn man die Beziehungen zwischen der Blutsenkung und der entwicklunghemmenden Wirkung des Vollbluts verfolgt, so neigt Gruppe I zum ganz parallelen Verlauf beider Reaktionen, was jedoch in der zweiten und der dritten Gruppe nicht besonders zu bemerken

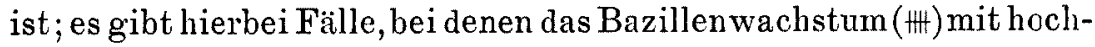
gradiger Beschleunigung der Blutsenkung auftritt.

Also zeigen, mit Ausnahme der Gruppe I, alle einen Prozentsatz, nach dem das Bazillenwachstum von der Blutsenkungsgeschwindigkeit wenig beeinflusst wird. „Dynamisch" ist, wie Tabelle I und II zeigen, bei den gebesserten Fällen das Verschwinden der entwicklunghemmenden Wirkung des Vollbluts meistens von Verminderung der Blutsenkunggeschwindigkeit begleitet, aber bei den sterbenden Fällen ist das Verhältnis meistens umgekehrt.

5. Beziehungen zwischen der entwicklunghemmenden Wirkung des Vollbluts auf Tuberkelbazillen und der Komplementbindungsreaktion (Kbg. R.)

Über die Zeit, wo nach tuberkulöser Infektion die Kbg. R. auftritt, herrscht noch keine feste Ansicht. Anch in zeitlicher Beziehung bestehen zwischen dem Auftreten der Tuberkulinallergie und der Kbg: R. individuelle Verschiedenheiten. Dies beruht wahrscheinlich auf der verschieden grossen Menge der Infektionsbazillen, der Herstellungsweise des Antigens und des dafür verwandten Bazillenstamms usw. 
Es gibt sogar, wie schon Me y e ${ }^{28)}$ und Sas a ki berichteten, Fälle, wo die Kbg. R. im ganzen Verlauf der Tuberkulose kein einziges Mal auftritt. Aber nach unserer Erfahrung tritt die Kbg. R. im allgemeinen später als die Tuberkulinallergie auf ; nur bei wenigen Fällen, bei denen während mehrerer Monate nach der Infektion keine Tuberkulinallergie auftritt, ist das Verhältnis umgekehrt.

Die Beziehungen zwischen dem Verlauf der Tuberkulose und der Kbg. R. sind schon, wie oben erwähnt, von Sasaki, Kumagai und Ozeki in unserer Klinik behandelt worden. Danach verschwindet, wenn die Krankheit zur Heilung neigt, die Komplementbindungsreaktion gewöhnlich früher als die Tuberkulinallergie, wogegen bei den Fällen, die eine Wendung zum Tode nehmen, erst die Tuberkulinallergie und dann die Kbg. R. verschwindet. Was die Beziehungen zwischen der entwicklunghemmenden Wirkung des Vollbluts auf Tuberkelbazillen und der Kbg. $R$. betrifft, so ist von vorn herein zu vermuten, dass beide Reaktionen in innigem Verhältnis zueinander stehen, da sie beide im Blut auftretende Antikörper sind.

Aus Tabelle VII ersieht man, dass von der Kbg. R. bei der Gruppe I (-) $39(75 \%),(+) 12(23 \%),(+t) 1(1,9 \%)$, (H+ und (卅) 0 betragen, dagegen in der Gruppe II (-) $99(43,6 \%),(+) 62(27,7 \%)$, (t+) 26 (11,4\%), (H) 16 (7\%) und (HH) $24(10,5 \%)$, d.h. die stark positiven Fälle nehmen allmällich zu. Aber in Gruppe III betragen von der Kbg. R. (-) $25(43,1 \%),(+) 15(25,8 \%),(+t)$ und (+⿻一l) je $8(13,8 \%)$ und (H) $2(34 \%)$. Das bedeutet, dass die Kbg. R. mit fortschreitender Tuberkulose allmählich ihre positive Zahl vermehrt und bei herannahendem Tode vermindert.

Was die Beziehungen zwischen der entwicklunghemmenden Wirkung des Vollbluts auf Tuberkelbazillen und der Kbg. R. betrifft, so sieht man, dass bei Gruppe I die hemmende Wirkung fortwährend parallel mit der Kbg. R. verläuft. Dies tritt bei Gruppe II und III und bei der Gesamtzahl noch dentlicher zutage. Anderseits gibt es als Ausnahmefälle bei Gruppe II allerdings 3 Fälle $(12,5 \%)$ und bei

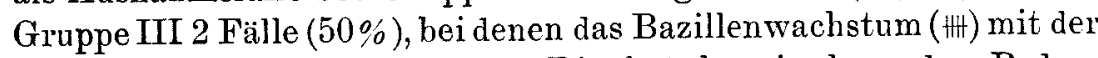
Kbg. R. (州) vergesellschaftet ist. Dies hat aber eine besondere Bedeutung, denn bei den ersten 3 Fällen neigt die Krankheit zur Heilung, aber ihre Kbg. R. verweilt noch in (페), während das Bazillenwachstum lebhafter geworden ist, und bei den letzten 2 Fällen ist die Krankheit ins Endstadium eingetreten, so dass das Bazillenwachstum leb-

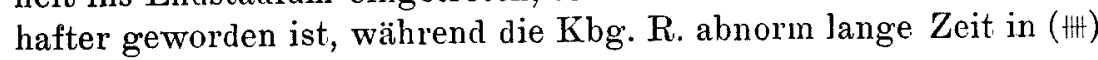
verweilt hat. 
Tabelle VII.

Eutwicklunghemmende Wirkung und Komplementbindungsreaktion.

\begin{tabular}{|c|c|c|c|c|c|}
\hline \multirow{3}{*}{$\begin{array}{l}\text { Bazillen- } \\
\text { wachstum }\end{array}$} & \multicolumn{5}{|c|}{$\begin{array}{c}\text { Gruppe I } \\
\text { Primärinfektion, Pleuritis und Peritonitis }\end{array}$} \\
\hline & \multicolumn{5}{|c|}{ Kbg. R. } \\
\hline & $(-)$ & $(+)$ & $(t+)$ & $(H)$ & $($ (WH) \\
\hline $\begin{array}{c}(+\ldots t) \\
(H t) \\
(+t) \\
(+) \\
(-) \\
\text { Gesamtzalıl }\end{array}$ & 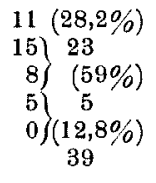 & $\begin{array}{lc}1 & (8,3 \%) \\
5 & 8 \\
3\} & (66,6 \%) \\
2 & 3 \\
1\} & (25 \%) \\
& 12\end{array}$ & $\left.\begin{array}{c}0 \\
0 \\
1 \\
6\end{array}\right\} \begin{array}{c}1 \\
(100 \%) \\
1\end{array}$ & $\begin{array}{l}0 \\
0 \\
0 \\
0\end{array}$ & $\begin{array}{l}0 \\
0 \\
0 \\
0\end{array}$ \\
\hline \multirow{3}{*}{$\begin{array}{l}\text { Bazillen- } \\
\text { wachstum }\end{array}$} & \multicolumn{5}{|c|}{$\begin{array}{l}\text { Gruppe II } \\
\text { Frühinfiltrat und Tuberculosis pulmonum (gebessert) }\end{array}$} \\
\hline & \multicolumn{5}{|c|}{ Kbg. R. } \\
\hline & $(-)$ & $(t)$ & $(t+)$ & $(H)$ & $($ HWI \\
\hline $\begin{array}{c}(+11) \\
(+t+) \\
(t+) \\
(+) \\
\text { GesamtzahI }\end{array}$ & $\begin{array}{l}10 \quad(10 \%) \\
26\} \quad 53 \\
27\}(53,3 \%) \\
21\{36 \\
15\}(36,3 \%) \\
99\end{array}$ & 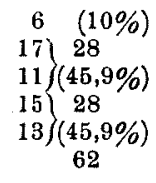 & 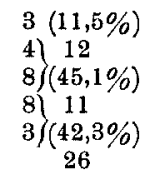 & $\left.\begin{array}{l}3 \\
3\} \\
4\} \\
6 \\
6 \\
3\end{array}\right\} \begin{array}{c}73,7 \%) \\
9 \\
(56,2 \%) \\
16\end{array}$ & $\begin{array}{l}3(12,5 \%) \\
3\} 8 \\
5\}(66,6 \%) \\
5\left\{\begin{array}{c}13 \\
8\}(54,1 \%) \\
24\end{array}\right.\end{array}$ \\
\hline \multirow{3}{*}{$\begin{array}{l}\text { Bazillen- } \\
\text { wachstum }\end{array}$} & \multicolumn{5}{|c|}{$\begin{array}{c}\text { Gruppe III } \\
\text { Tuberculosis pulmonum (gestorben) }\end{array}$} \\
\hline & \multicolumn{5}{|c|}{ Kbg. R. } \\
\hline & $(-)$ & $(+)$ & $(t+)$ & $(H+1)$ & (HAt) \\
\hline $\begin{array}{c}(+4) \\
(+t t) \\
(+t) \\
(+) \\
(-) \\
\text { Gesamtzahl }\end{array}$ & $\begin{array}{ll}6 & (24 \%) \\
8) & 12 \\
4\} & (48 \%) \\
3) & 7 \\
4 & (28 \%) \\
& 25\end{array}$ & $\begin{array}{l}2(13,3 \%) \\
3) \quad 5 \\
2\}(33,3 \%) \\
4) \quad 8 \\
4\}(53,3 \%) \\
15\end{array}$ & \begin{tabular}{l|l} 
& 0 \\
2 & 2 \\
$0\}$ & $(25 \%)$ \\
4 & 6 \\
2 & $(75 \%)$ \\
& 8
\end{tabular} & 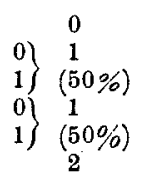 & $\begin{array}{c}2 \quad(25 \%) \\
0 \\
1) \\
5 \\
(75 \%) \\
8\end{array}$ \\
\hline \multirow{3}{*}{$\begin{array}{l}\text { Bazillen- } \\
\text { wachstum }\end{array}$} & \multicolumn{5}{|c|}{ Gesamtfälle } \\
\hline & \multicolumn{5}{|c|}{ Kbg. R. } \\
\hline & $(-)$ & $(+)$ & $(t+)$ & $(H+)$ & (HI) \\
\hline $\begin{array}{c}(H+) \\
(H+) \\
(+t) \\
(+) \\
(-) \\
\text { Gesamtzahl }\end{array}$ & $\begin{array}{c}27(16,5 \%) \\
49\} 88 \\
39\}(54 \%) \\
29\} 48 \\
19\}(29,4 \%) \\
163\end{array}$ & $\begin{array}{c}9(10,1 \%) \\
25)(41 \\
16)(46,6 \%) \\
21\} 39 \\
18\}(43,8 \%) \\
89\end{array}$ & 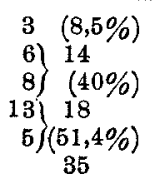 & $\begin{array}{l}\left.\begin{array}{c}0 \\
3 \\
5 \\
5\end{array}\right\}\left(\begin{array}{c}8,4 \%) \\
6 \\
40 \\
4\end{array}\right\}(55,5 \%) \\
18\end{array}$ & $\begin{array}{c}5(15,6 \%) \\
3) 8 \\
5\}(25 \%) \\
6\} 19 \\
13\}(59,3 \%) \\
32\end{array}$ \\
\hline
\end{tabular}




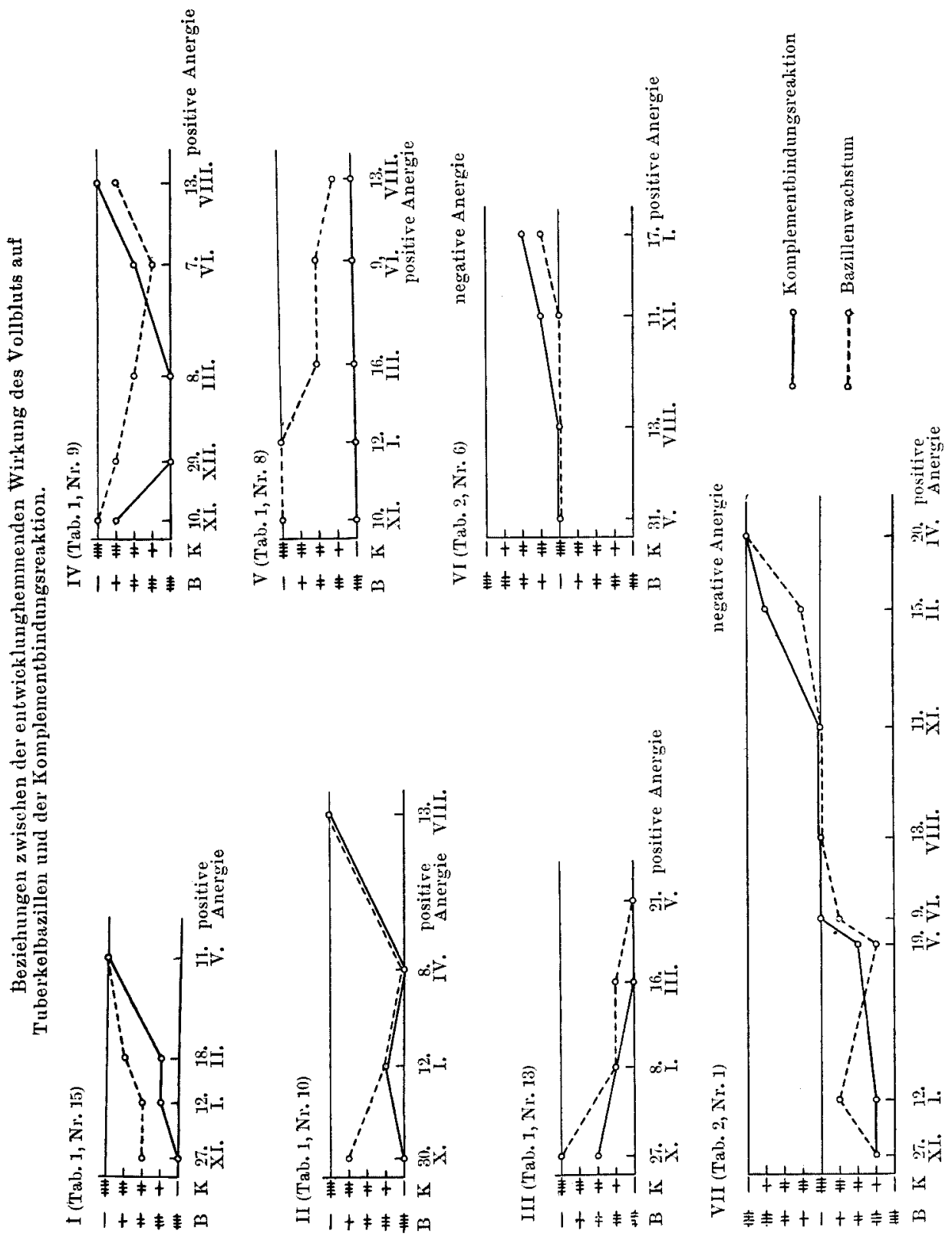


Bei Gruppe I in Tabelle VII zeigt sich, dass die entwicklunghemmende Wirkung auf Tuberkelbazillen gewöhnlich früher als die $\mathrm{Kbg}$. R. auftritt, schon bei $28(7,8 \%)$ unter den Fällen mit einer Kbg. R. von (-) ist das Bazillenwachstum mehr oder weniger gehemmt. Wie die Fälle Nr. 13, 16, 17 u. 19 in Tabelle I zeigen, verschwindet die entwicklunghemmende Wirkung etwas später als die Kbg. R., wenn sich die Tuberkulose zur Heilung wendet, aber bei den gestorbenen Fällen, z. B. Fall Nr. 1, 2 u. 4 in Tabelle II, verschwindet zuerst die Kbg. R. und dann die entwicklunghemmende Wirkung. Das kommt daher, dass das Verschwinden der Kbg. R. bei der Heilung zum Aufhören der Antikörperbildung infolge der Vermindernng des Antigens führt, so dass die entwicklunghemmende Wirkung dazu neigt, nach dem Schwinden der Kbg. R. allmählich auch zu verschwinden, wogegen das Verschwinden der Kbg. R. im Endstadium zur Verminderung der Antikörperneubildung führt, obwohl das Antigen, d. h. die Mikroorganismen, immer mehr zunimmt, so dass die entwicklunghemmende Wirkung dazu neigt, nach dem Schwinden der Kbg. R. relativ schnell abzunehmen.

Durch Kurvendarstellung des Verlaufs der entwicklunghemmenden Wirkung des Vollbluts und der Kbg. R. bei einigen Fällen werden die Beziehungen zwischen beiden sehr klar. (S. Kurven in der vorigen Seite.)

Hieraus lässt sich erkennen, dass die entwicklunghemmende Wirkung des Vollbluts auf Tuberkelbazillen und die Kbg. R. immer parallel verlaufen. $\mathrm{Zu}$ bemerken ist noch, dass das Wachstum der Tuberkelbazillen, wie schon Meyer und Sasaki berichteten, z. B. bei den, Fällen Nr. 8, 19, 20 usw. in Tabelle I, wo die Kbg. R. immer negativ bleibt, ziemlich stark gehemmt wird.

Dies weist darauf hin, dass auch das blut entwicklunghemmendi wirkt, in welchem die Ambozeptoren der Komplementbindung noch. nicht aufgetreten sind. Und hierbei ist auch zu berücksichtigen, dassdas benutzte Antigen mit dem S-Stamm humaner Tuberkelbazillen. hergestellt worden war, so dass die Kbg. $\mathrm{R}$. in diesem Blut vielleicht positiv ausgefallen wäre, wenn wir einen anderen Stamm als Antigen. benutzt hätten. Jedenfalls gehen die entwicklunghemmende Wirkung des Vollbluts auf Tuberkelbazillen und die Kbg. R. immer parallel, und man muss sagen, dass beide in einem sehr innigen Verhältnis zueinander stehen. 


\section{Beziehungen zwischen der entwicklunghemmen- den Wirkung auf Tuberkelbazillen und der Leukozytenzahl.}

Die Leukozytenzahl Gesunder beträgt nach Blumenfeld, ${ }^{99}$ Eicke ${ }^{30)}$ Glöel, ${ }^{31)}$ Gutzeit ${ }^{322}$ u. a. 400-10000. Sie bleibt auch bei Tuberkulösen ungefähr innerhalb derselben Grenzen, nimmt aber bis 11000 oder noch mehr zu, wie Steffe ${ }^{333}$ Decastello $0^{34}$ u. a. bemerken, wenn die Tubeakulose schwer wird, und beim Lebensende soll sie 15000-20000 betragen können.

Bei der Gruppe I in Tabelle VIII zeigt die Leukozytenzahl bei 51 unter 52 Fällen einen normalen Wert, während sie sich bei 17 Fällen der Gruppe II $(7,4 \%)$ auf $10000-12000$ und bei $3(1,3 \%)$ auf über 12000 vermehrt hat. In Gruppe III beträgt die Leukozytenzahl bei $21(36,2 \%) 10000-12000$ und bei $8(13,8 \%)$ über 12000 ; also ist insgesamt bei $29(50 \%)$ Leukozy tose vorhanden. Dies zeigt die Neigung. zur Leukozytose bei schwerer Tuberkulose, was oben schon erwähnt worden ist. Wright vertritt die Ansicht, dass die entwicklunghemmende Wirkung des Vollbluts von der Phagozytose der Leukozyten herrührt, aber Sato u. a. behaupten dagegen, dass sie gar keinen $\mathrm{Zu}$ sammenhang damit hat.

Wenn man die Beziehungen zwischen der entwicklunghemmenden Wirkung des Vollbluts auf Tuberkelbazillen und der Leukozytenzahl verfolgt, so zeigt die Gruppe I kein deutliches einheitliches Verhältnis, bei den Gruppen II und III dagegen verlaufen beide im allgemeinen parallel. Aber bei der Gruppe III, in der die Leukozyten über 10000 zählen, wird das Bazillenwachstum wieder lebhafter.

Also scheinen im ganzen die entwicklunghemmende Wirkung des Vollbluts auf Tuberkelbazillen und die Leukozytenzahl immer parallel zu verlaufen. Dagegen sind bei ,dynamischer" Betrachtung der Ergebnisse, z. B. bei den Fällen Nr. 7 u. 16 in Tabelle I und Nr. 3, 5 u. 7 in Tabelle II, die Beziehungen zwischen beiden scheinbar nicht so innig.

7. Beziehungen zwischen der entwicklunghemmenden Wirkung des Vollbluts auf Tuberkelbazillen und den Lymphozyten.

Die Lymphozytenzahl Gesunder beträgt nach Blumenfeld 20- 


\section{Tabelle VIII.}

Entwicklunghemmende Wirkung und Leukozyten.

\begin{tabular}{|c|c|c|c|c|c|}
\hline \multirow{3}{*}{$\begin{array}{l}\text { Bazillen- } \\
\text { wachstum }\end{array}$} & \multicolumn{5}{|c|}{$\begin{array}{c}\text { Gruppe I } \\
\text { Primärinfektion, Plenritis und Peritonitis }\end{array}$} \\
\hline & \multicolumn{5}{|c|}{ Leukozyten } \\
\hline & -4000 & $\begin{array}{l}4000- \\
7000\end{array}$ & $\begin{array}{l}7000- \\
10000\end{array}$ & $\begin{array}{l}10000 \\
12000\end{array}$ & über 12000 \\
\hline $\begin{array}{c}(H+) \\
(H+) \\
(t+) \\
(+) \\
(-) \\
\text { Gesamtzahl }\end{array}$ & $\begin{array}{c}0 \\
0\}(100 \%) \\
0 \\
1\end{array}$ & $\begin{array}{ll}4 & (21 \%) \\
6 & 11 \\
5\} & (58 \%) \\
4) & 4 \\
0\} & (21 \%) \\
& 19\end{array}$ & 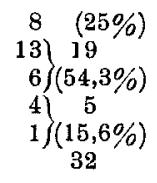 & $\begin{array}{l}0 \\
0 \\
0 \\
0\end{array}$ & $\begin{array}{l}0 \\
0 \\
0 \\
0\end{array}$ \\
\hline \multirow{3}{*}{$\begin{array}{l}\text { Bazillen- } \\
\text { wachstum }\end{array}$} & \multicolumn{5}{|c|}{$\begin{array}{l}\text { Gruppe II } \\
\text { Frähinfiltrat und Tuberculosis pulmonum (gebessert) }\end{array}$} \\
\hline & \multicolumn{5}{|c|}{ Leukozyten } \\
\hline & -4000 & $\begin{array}{l}4000- \\
7000\end{array}$ & $\begin{array}{l}7000- \\
10000\end{array}$ & $\begin{array}{l}10000 \\
12000\end{array}$ & über 12000 \\
\hline $\begin{array}{c}(+H t) \\
(+H) \\
(++) \\
(+) \\
(-) \\
\text { Gesamtzahl }\end{array}$ & $\begin{array}{ll} & 0 \\
0) & 1 \\
1\} & (50 \%) \\
1\} & 1 \\
0\} & (50 \%) \\
2 & \end{array}$ & $\begin{array}{c}8(10,1 \%) \\
21) 40 \\
19\}(50,6 \%) \\
18\} 31 \\
13\}(39,2 \%) \\
79\end{array}$ & $\begin{array}{l}12(9,5 \%) \\
27) 58 \\
31\}(46 \%) \\
29\} 56 \\
27\}(44,4 \%) \\
\begin{array}{c}126 \\
\end{array}\end{array}$ & 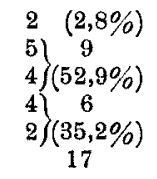 & $\begin{array}{l}0 \\
0 \\
0 \\
0\end{array}$ \\
\hline \multirow{3}{*}{$\begin{array}{l}\text { Bazillen- } \\
\text { wachstum }\end{array}$} & \multicolumn{5}{|c|}{$\begin{array}{c}\text { Grappe III } \\
\text { Tuberculosis pulmonum (gestorben) }\end{array}$} \\
\hline & \multicolumn{5}{|c|}{ Leukozyten } \\
\hline & -4000 & $\begin{array}{l}4000- \\
7000\end{array}$ & $\begin{array}{l}7000- \\
10000\end{array}$ & $\begin{array}{l}10000- \\
12000\end{array}$ & über 12000 \\
\hline $\begin{array}{c}(H+) \\
(H t) \\
(t+) \\
(+) \\
(-) \\
\text { Gesamtzahl }\end{array}$ & $\begin{array}{l}0 \\
0 \\
0 \\
0\end{array}$ & $\begin{array}{ll}3 & (30 \%) \\
2\} & 3 \\
1\} & (30 \%) \\
2\} & 4 \\
2\} & (40 \%) \\
& 10\end{array}$ & $\begin{array}{l}3(15,7 \%) \\
7)(12 \\
5\}(63,1 \%) \\
1) \begin{array}{l}4 \\
3\} \\
(21 \%) \\
19\end{array}\end{array}$ & $\begin{array}{cc}1 & (5 \%) \\
3) & 4 \\
1\} & (19 \%) \\
6) & 16 \\
10 & (76,1 \%) \\
& \\
21 & \end{array}$ & 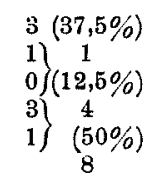 \\
\hline \multirow{2}{*}{$\begin{array}{l}\text { Bazillen- } \\
\text { wachstum }\end{array}$} & \multicolumn{5}{|c|}{ Leukozyten } \\
\hline & -4000 & $\begin{array}{l}4000- \\
7000\end{array}$ & $\begin{array}{l}7000- \\
10000\end{array}$ & $\begin{array}{l}10000- \\
12000\end{array}$ & tiber 12000 \\
\hline $\begin{array}{c}(+H) \\
(t+t) \\
(t+) \\
(+) \\
(-) \\
\text { Gesamtzahl }\end{array}$ & 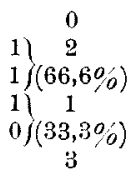 & $\begin{array}{l}15(13,9 \%) \\
29)(54 \\
25)(50 \%) \\
24) 39 \\
15)(33,3 \%) \\
\begin{array}{c}108 \\
108\end{array}\end{array}$ & 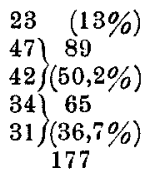 & $\begin{array}{l}3 \\
8\} 13 \\
5\}(34,1 \%) \\
10\left\{\begin{array}{c}22 \\
12\end{array}\right\}(57,9 \%) \\
38\end{array}$ & $\begin{array}{l}3(27,2 \%) \\
1) \quad 1 \\
0\} \quad(9 \%) \\
6) \quad 7 \\
1\}(63,6 \%) \\
11\end{array}$ \\
\hline
\end{tabular}


$28 \%$, aber nach Eicke und Steffen $25-30 \%$, im allgemeinen gilt ungefähr $20-35 \%$ als normale Zahl. Hermann, ${ }^{35)} \mathrm{Ku}$ sama, ${ }^{36)} \mathrm{Nae}-$ geli $^{3 z}$ u. a. berichteten, dass bei schwerer Tuberkulose die Lymphozyten bis auf $10 \%$ oder sogar noch weniger abnehmen. Deshalb berichteten $\mathrm{Naegeli}$, Sylla ${ }^{38}$ u. a., dass ihre Abnahme Verschlimmerung und ihre Zunahme Besserung der Tuberkulose anzeigen.

Wie man aus Tabelle I ersieht, gibt es bei Gruppe I keinen einzigen Fall, dessen Lymphozytenprozentsatz niedriger als $10 \%$ ist, aber $2(3,8 \%)$ zeigen $10-19 \%$ Lymphozyten. Bei Gruppe II gibt es zwar ebenfalls keinen Fall, dessen Lymphozy tenprozentsatz niedriger als $10 \%$ ist, aber $28(12,3 \%)$ zeigen $10-19 \%$. Aber in Gruppe III sind 2 Fälle $(3,4 \%)$, die weniger als $10 \%$ haben und $15(26 \%)$ mit $10-$ 19\%. Dies bestätigt die Ansicht obiger Autoren, dass die Abnahme der Lymphozyten prognostisch ein schlechtes Zeichen ist.

Verfolgt man die Beziehungen zwischen der entwicklunghemmenden Wirkung des Vollbluts auf Tuberkelbazillen und dem Lymphozytenprozentsatz, so findet man, dass bei Gruppe I die Fälle mit Bazillenwachstum (H) und die mit $(+)$ und $(-)$ unter den Fällen mit normaler Lymphozytenzahl gleich zahlreich sind. Unter den Fällen mit vermehrter $(19,4 \%)$ Lymphozytenzahl gibt es mehr Fälle mit Bazillenwachstum (冊) und weniger Fälle mit beschränktem Wachstum (+) und (-), nämlich $28,5 \%$ und $14,3 \%$.

Bei den Gruppen II und III vermehren sich sowohl die Fälle mit Bazillenwachstum (WI) als auch die mit (+) und (-) umso stärker, je kleiner die Lymphozytenzahl wird. Ebenso verhält es sich auch mit den gestorbenen Lungentuberkulösen.

Kurz zusammengefasst, die Fälle mit Bazillenwachstum (州) vermehren und die mit Bazillenwachstum $(+)$ und $(-)$ vermindern sich, wenn die Lymphozyten zunehmen; hingegen zeigt der Prozentsatz

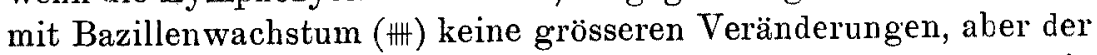
mit Bazillenwachstum $(+)$ und (-) auffallende Vermehrung, wenn die Lymphozyten abnehmen.

Denn durch Zunahme der Lymphozyten wird die entwicklunghemmende Wirkung des Vollbluts auf Tuberkelbazillen abgeschwächt und durch ihre Abnahme verstärkt. Mit andern Worten, wenn die Tuberkulose sich zur Heilung wendet, wobei sich die Lymphozyten meistens vermelren, neigt die entwicklunghemmende Wirkung des. Vollbluts auf Tuberkelbazillen zum Sinken, im umgekehrten Fall, wobei sich der Lymphozytenprozentsatz meistens vermindest, besteht 
Tabelle IX.

Entwicklunghemmende Wirkung nud Lymphozyten.

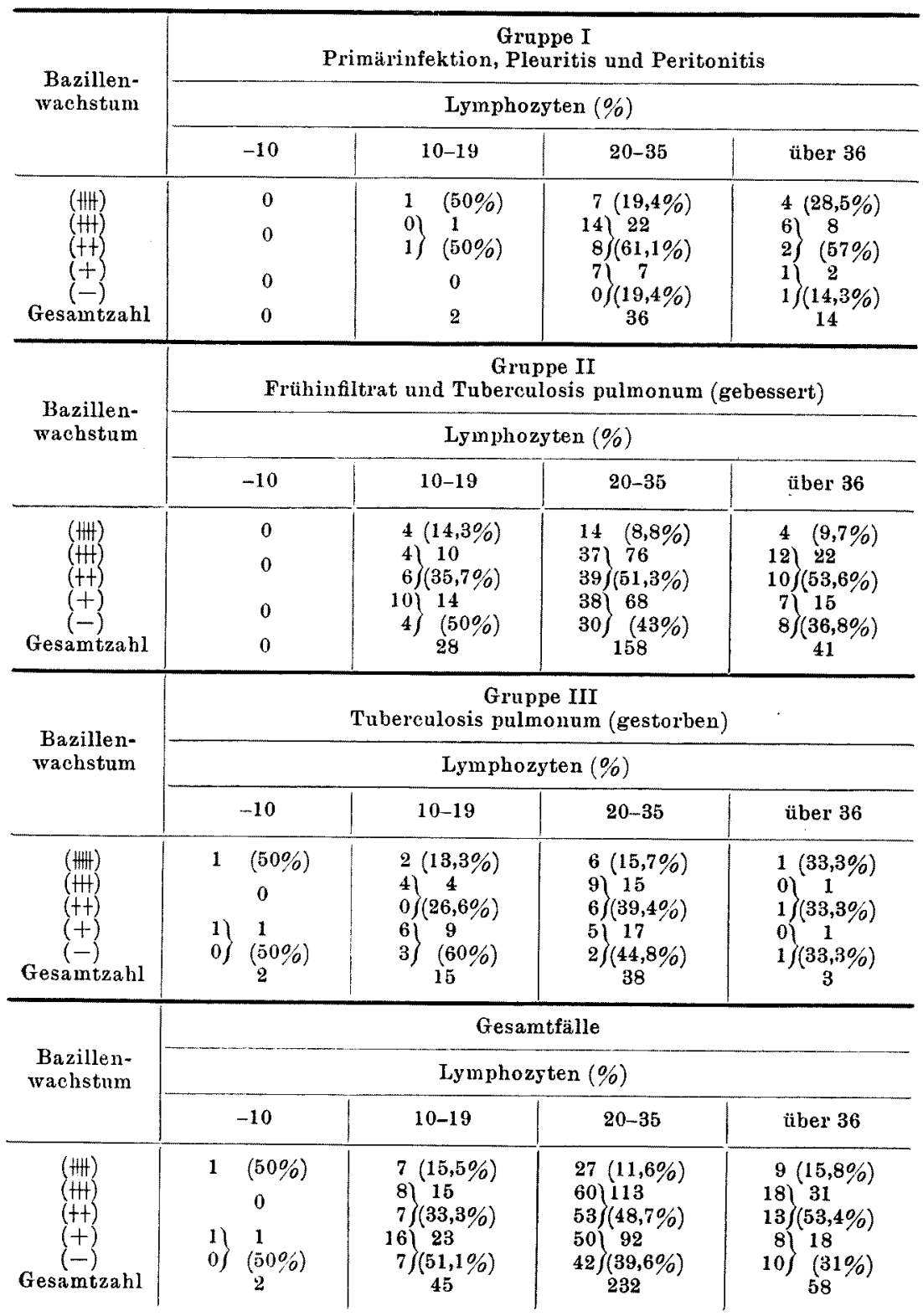


Neigung zu ihrem Steigen. Aber ,dynamisch“ übt die Veränderung des Lymphozytenprozentsatzes, wie aus vielen Fällen in Tabelle I und II ersichtlich, keinen grossen Einf̣uss aus.

\section{Zussammenfassung.}

1. Die entwicklunghemmende Wirkung des Vollbluts Tuberkulöser auf Tuberkelbazillen steht in einem gewissen Verhältnis zum Krankheitsstadium, zur Tuberkulinallergie, zur Senkungsreaktion der Blutkörperchen und zur Kbg. R., aber in nicht so innigem Verhältnis zur Körpertemperatur und Leukozyten- und Lymphozyten zahl.

2. Die entwicklunghemmende Wirkung des Vollblut auf Tuberkelbazillen neigt dazu, mit den fortschreitenden Krankheitsstadien immer parallel zu verlaufen und erst dann wieder schwächer zu werden, wenn die Tuberkulose sich zur Heilung oder zum Endstadium wendet.

3. Die entwicklunghemmende Wirkung des Vollbluts auf Tuberkelbazillen setzt gleichzeitig mit oder etwas später als das Auftreten der Tuberkulinallergie ein, und im weiteren Verlauf gehen beide meistens parallel, aber wenn die Tuberkulose sich zur Heilung wendet, wird die erste vor Abnahme der Tuberkulinallergie allmählich schwächer, und wenn sich die Tuberkulose dem Endstadium nähert, so nimmt die erste später ab, als die Tuberkulinallergie verschwindet.

4. Was die Beziehungen zwischen der entwicklunghemmenden Wirkung des Vollbluts auf Tuberkelbazillen und der Senkungsreaktion der Blutkörperchen betrifft, so neigt diese schon vor dem Auftreten jener zur Beschleunigung und geht dann im weiteren Verlauf der Tuberkulose mit ihr beinahe parallel; wenn aber die Tuberkulose sich zur Heilung wendet, nimmt diese bis zum normalen Wert ab, und jene wird dann etwas später auch schwächer. Aber umgekehrt sinkt diese, bevor jene zum normalen Wert zurückkehrt, wenn die Tuberkulose ins Endstadium eintritt.

5. Die entwicklunghemmende Wir'kung des Vollbluts Tuberkulöser auf Tuberkelbazillen und die Komplementbindungsreaktion stehen in ganz innigem Verhältnis zueinander, so dass sie in fast allen Fällen ganz parallel zu verlaufen scheinen. Die erste setzt vor dem 
Auftreten der letzten ein, aber im ganzen Verlauf der Tuberkulose geht sie mit ihr ganz parallel, und wenn die Tuberkulose sich zur Heilung wendet, vermindert sie sich etwas später, als die Kbg. R. verschwindet, und ebenso wenn die Tuberkulose sich dem Endstadium nähert.

Dies Kosten für diese Untersuchung sind von der HōonkaiStiftung bestritten worden, wofür ich hier den besten Dank ausspreche.

Prof. T. Kumagai.

\section{Literatur.}

(1) Wrig ht, Lancet, 1924, 206, 218.

(2) B a nner ma nn, Brit. Journ. of Exp. Pathol., 1927, 8, 209.

(3) Me is neer, Zentralbl. f. Bakt. Orig., 1928, 106, 210.

(4) Sonak, Zentralbl. f. Bakt. Orig., 1930, 115, 173.

(5) S a to, Jikken Igaku Zasshi, 1926, 10, 871.

(6) I to, Kekkaku, 1930, 8, 291.

(7) Ogata u. Schibukawa, Kekkaku, 1932, 10, 247.

(8) Ima mura u. Schibukawa, Kekkaku, 1933, 11, 209.

(9) Sehibukawa, Kekkaku, 1983, 11, 63.

(10) Ogata, Kekkaku, 1932, 10, 117.

(11) Sas a ki, Tohoku Jl. of Exp. Med., 1933, 20, 230.

(12) K n magai u. Ozeki, Shindan to Chiryo, Ergänzungsband, 1933; 230.

(13) S u z ki, Tohoku Jl. of Exp. Med., 1934, 23, 79

(14) Ma rmorek, Zeitschr. Tbk., 1900, 1, 1444.

(15) S pengler, Wien. med. Wschr., 1902, 658.

(16) Much, Beitr. Klin. Tbk., 8, 85.

(17) Kn o11, Beitr. Ḱlin. Tuk., 1910, 211.

(18) K a h n, Amer. Rev. Tbc., 1928, 18, 630.

(19) S panier, Zbl. f. Bakt. 1. Orig., 1933, 127, 477.

(20) Jütten u. Ha armann, Münch. med. Wscht., 1920, 692.

(21) Ku maga i, Nippon Naikagakkai Zasshi, 1934, 21, 1.

(22) Alvarey u. Barralt, Zbl. f. Gesammt-Tbk, forseh., 1926, 25, 48.

(23) We stergren, Ergeb. d. inn. Med. u. Kinderh., 1924, 26, 577.

(24) Katz u. Leffk owitz, Ergeb. d. inn. Med. u. Kinderh., 1928, 33, 266.

(25) Katsunu m a, Kekkaku, 1934, 12, 45i.

(26) Oka be, Tohoku Igaku Zasshi, 1934, 17, 142.

(27) S pies, Beitr. Klin. Tbk., 1923, 56, 67.

(28) Me ger, Berl. kl. Wschr., 1910, 2265.

(29) Blumenfeld, Ztschr. f. exper. Path., 1910, 20, 14.

(30) Eicke, Beitr. Klin. Tbk., 1923, 56, 669.

(31) Glöel, Beitr. Klìn. Tbk., 1920, 45, 404.

(32) Gutzeit, Wien. med. Wschr., 1931, 1270. 
(33) Steffen, Dtsch. Arch. kl. Ked., 1910, 98, 355.

(34) Decastello, Wien. med. Wschr., 1919, 669.

(35) Hermann, Ztschr. f. Tbk., 1916, 26, 336.

(36) K us a ma, Nippon Naikagakkai Zasshi, 1930, 4, 506.

(37) Na ge li, Blutkrankheiten u. Blutdiagnostik, Berlin 1931, 5. Aufl., 44.

(38) S glla, Med. Klin., 1932, 686. 